\title{
Formation and Collision of Multistability-Enabled Composite Dissipative Kerr Solitons
}

\author{
Wenle Weng, ${ }^{*}$ Romain Bouchand, and Tobias J. Kippenberg ${ }^{\dagger}$ \\ Swiss Federal Institute of Technology in Lausanne (EPFL), CH-1015 Lausanne, Switzerland
}

(Received 13 May 2019; revised manuscript received 24 December 2019; accepted 19 February 2020; published 23 April 2020)

\begin{abstract}
Multistability in Kerr resonators which are driven by continuous or modulated optical waves gives rise to the superposition of distinct nonlinear states, yielding a unique platform for studying complex soliton dynamics. Here, by pumping a crystalline microresonator with two lasers that are frequency detuned from each other by one or multiple cavity free spectral ranges, we go beyond the traditional bichromatic pumping framework and enter an unexplored multistability regime that allows observing novel dynamics including composite solitons and successive soliton collisions. We generate complex frequency comb patterns, observing the velocity mismatch between the solitons and the dual-pumping-induced lattice traps and showing the synchronization of the repetition rates of constituent distinct solitons under the influence of index-barrier-induced intersoliton repulsion. We also demonstrate soliton collisions and observe transient soliton response with spectral analysis and ultrafast imaging, highlighting the eigenfrequency of dissipative soliton dynamics that coincides the "soliton $(\mathcal{S})$ resonance." Furthermore, we exploit the higher-order dispersion effect to manipulate the intrinsic group velocity mismatch between distinct solitons and demonstrate reversible switching between the composite soliton state and the soliton collisional state. Our findings bring to light the rich physics of the Kerr multistability and may equally be useful in microcombbased spectroscopy and metrology.
\end{abstract}

DOI: 10.1103/PhysRevX.10.021017

\section{INTRODUCTION}

Optical temporal solitons, particlelike optical wave packets that maintain their shape during propagation, have been extensively studied in fibers and lasers [1,2], owing to both the fascinating physics they present and the promise in optical communication, which however has not materialized due the potential cost of infrastructures that are compatible with wavelength division multiplexing. Dissipative solitons, which rely on a double balance of gain and loss as well as nonlinearity and dispersion, are however routinely used in mode-locked lasers. In recent years, a particular type of cavity solitons-temporal solitons in dissipative systems [3] with Kerr nonlinearity, also known as dissipative Kerr solitons (DKSs) - have been attracting surging research interest in platforms ranging from bulk free-space cavities [4] and fiber ring resonators [5] to microresonators of a variety of host materials [6,7]. In particular, DKSs generated in microresonators are, on the one hand, promising for numerous applications

\footnotetext{
*wenle.weng@epfl.ch

tobias.kippenberg@epfl.ch
}

Published by the American Physical Society under the terms of the Creative Commons Attribution 4.0 International license. Further distribution of this work must maintain attribution to the author(s) and the published article's title, journal citation, and DOI.
Subject Areas: Nonlinear Dynamics, Optics, Photonics

including spectroscopy $[8,9]$, low-noise microwave generation [10,11], imaging [12], and telecommunication [13] as they produce miniaturized and coherent frequency combs (microcombs). On the other hand, DKSs exhibit a plethora of interesting phenomena such as Stokes solitons [14], soliton breathers [15-17], and soliton crystals [18]. Yet, to date, direct soliton interactions, including shortrange binding [19,20] and collision [21,22], have not been thoroughly investigated in DKSs, despite the fact that they hold critical importance, not only for understanding the fundamental soliton dynamics, but also for applications such as a vernier spectrometer using counterpropagating DKSs [23], as well as tricomb spectroscopy [24] with spatial multiplexing of DKSs [25]. The difficulty is threefold: first, solitons pumped by the same lasers have the same group velocity, which makes the control of the relative locations of solitons difficult; second, because of the low output power and the high repetition rate of microresonator DKSs, commonly employed imaging techniques including dispersive Fourier transformation technique [26] and electro-optic imaging technique [27] cannot be applied to image the close interaction of similar DKSs due to the limited temporal window or the coarse resolution; third, because of internal disturbances such as mode crossings [28], DKSs usually interact with other DKSs via long-range dispersive-wave-mediated effects [29-32], forming groups with large intersoliton separations, thus prohibiting the inception of direct binding and collision. 
(a)

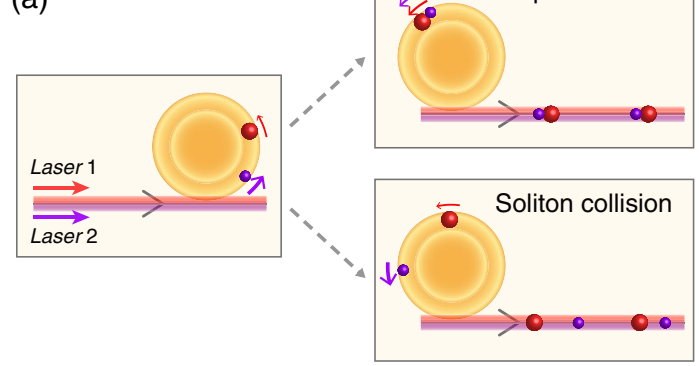

(b)

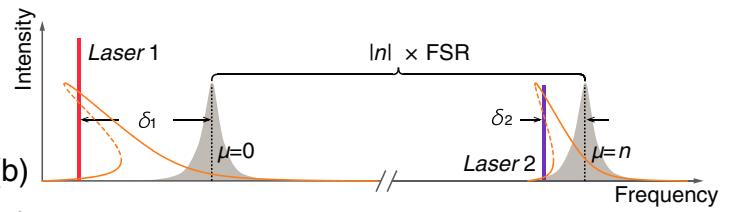

(c)

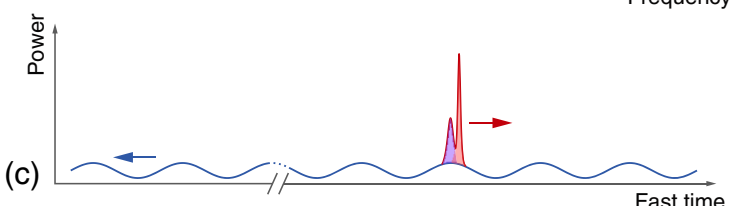

(d)

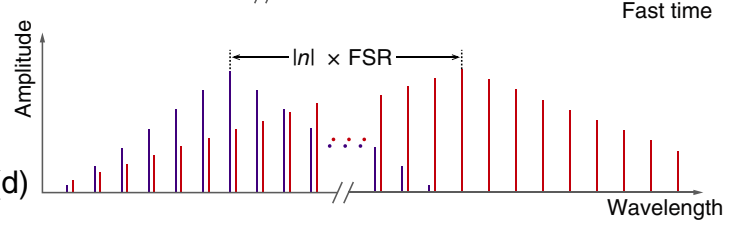

(e)

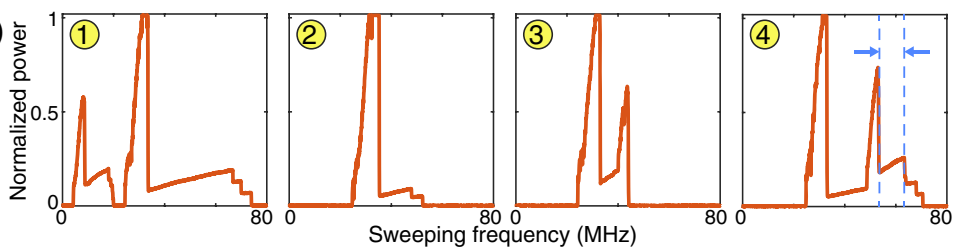

(f)

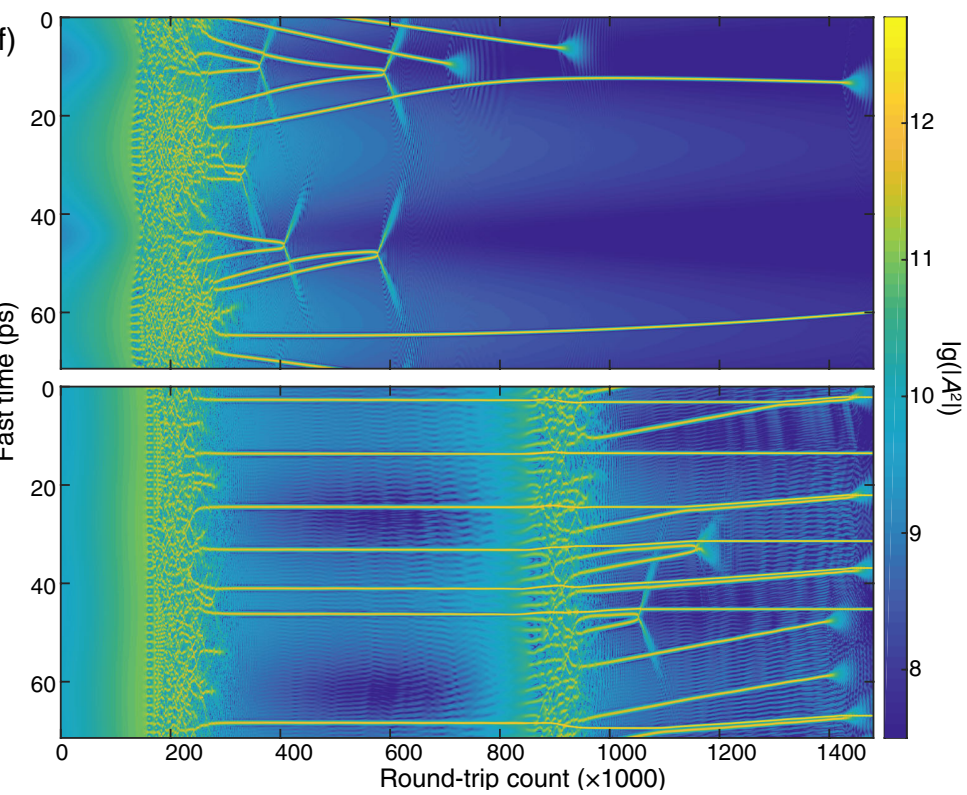

FIG. 1. Multistability dynamics in a dual-pumped Kerr resonator. (a) An illustration of the two possible dynamic states enabled by bichromatically pumped multistability, namely the composite soliton and the soliton collision. (b) The concept of the bichromaticpumping-enabled multistability in the frequency domain. (c) In the time domain, the two solitons (bound in the figure) travel at a velocity different from that of the intracavity lattice traps. (d) The two microcombs which correspond to the two distinct DKSs. (e) Experimentally generated comb power as the two lasers are swept across the resonances with a detuning difference $\Omega$. The four labeled panels respectively correspond to (1) $\Omega / 2 \pi=-20 \mathrm{MHz}$, both the soliton steps driven by the two lasers are shown separately, (2) $\Omega / 2 \pi=0 \mathrm{MHz}$, only one soliton state exists, (3) $\Omega / 2 \pi=10 \mathrm{MHz}$, the primary-pumped soliton state disappears after the secondary pump is coupled in efficiently, (4) $\Omega / 2 \pi=20 \mathrm{MHz}$, the coexistence of distinct soliton steps shows between the dashed lines. (f) The simulated intracavity intensity evolutions with conventional dual-pumped soliton lattice traps (upper panel, $\delta_{1}=\delta_{2}$ ) and bichromaticpumping-enabled multistability (lower panel, $\delta_{1}-\delta_{2}=70 \kappa$ ). The relative drifting of the DKS driven by the secondary laser in the lower panel is a result of the different intrinsic soliton group velocities due to the FSR difference between the two pumped resonances. The secondary solitons annihilate close to the end of the simulation because the effective detuning reaches the end of the secondary soliton existence range, which is much narrower than the primary soliton existence range due to the lower pump power.

Prior works used injected potential wells to trap multiple solitons together, which led to merging or annihilation of solitons [33-36], but, in general, the generation and the flexible control of close soliton binding and soliton collision are extremely challenging, and have not been studied experimentally.

In this work, by simultaneously pumping two optical resonances in the same mode family of a microresonator, we demonstrate that each resonance can be independently driven to its upper or lower stable branch of Kerr bistability $[37,38]$. As a result, the system can be operated in the regime of multistability, where multiple stable equilibrium states are allowed [39-42]. This bichromatically pumped multistability allows us to deterministically generate soliton collisions as well as composite solitons [see Fig. 1(a) for conceptual illustrations], which consist of two different solitons that mutually trap each other and form a stable soliton bound state [43]. Owing to the nonoverlapping comb spectra of different solitons, with an electro-optic sampling technique we are able to unveil not only the subpicosecond intersoliton separations in a composite soliton state, but also the eigenfrequencies of DKS dynamics when a collision event occurs. Moreover, by tuning the intrinsic group velocity difference between two dissimilar DKSs, we switch between the composite soliton state and a collisional state (characterized by periodic DKS collisions) in a reversible manner without soliton merging or annihilation.

\section{BICHROMATICALLY DRIVEN MULTISTABILITY}

Figures 1(b)-1(d) illustrate the concept of bichromaticpumping-enabled multistability. Two resonances in the same mode family are pumped by two laser fields with 
respectively controlled pump-resonance detunings $\delta_{1}$ and $\delta_{2}$. When the mismatch between the two detunings is relatively small, the beating of the laser fields creates potential wells on the amplitude or phase-modulated intracavity field, which are essentially lattice traps that can trap solitons and discipline the soliton group velocity (repetition rate) $[34,44,45]$. Similar situations have also been demonstrated with a pulse pumping scheme [45,46], although the lattice traps are created by multiple, rather than two, pumping laser fields. Once the detuning mismatch exceeds the maximum trapping range such that solitons can no longer follow the lattice traps, they drift to a location where the amplitude of the modulated intracavity field is below the critical point for soliton existence, leading to soliton annihilation [45]. Until now, the bichromatically pumped system has been operated in the well-known bistability regime [47-49] where only one DKS species can be generated. However, as the detuning mismatch continues to increase, the bistability paradigm fails, and the system is admitted to the multistability regime, where both resonances can be pumped to the upper branches of the Kerr bistability [see Fig. 1(b)], supporting their own DKS. In this situation, in time domain, the intracavity field shows the coexistence of two distinct DKS species and the lattice traps that are drifting with a different velocity [see Fig. 1(c)]. One should note that this bichromatically driven multistability is physically distinct from the multistability regime driven in a single resonance by a modulated laser [41], as the modulated light source in the earlier work could not create soliton lattice traps with intracavity potential wells. In contrast, here the solitons are continuously drifting over the minima and maxima of the lattice traps, yet they are able to survive. In the frequency domain such a coexistence of solitons generates two sets of microcombs mutually interleaved [see Fig. 1(d)].

Figure 1(e) shows the experimentally generated microcomb power when a primary laser $(\sim 400 \mathrm{~mW})$ and a secondary laser $(\sim 100 \mathrm{~mW})$ are coupled into a magnesium fluoride $\left(\mathrm{MgF}_{2}\right)$ microresonator with a free spectral range (FSR) of $14.09 \mathrm{GHz}$. With the total pump power of $\sim 500 \mathrm{~mW}$ from an erbium-doped fiber amplifier (EDFA) that is used to amplify both the primary and the secondary lasers, this power ratio is suitable for achieving a maximum soliton coexistence range. The frequency separation between the two lasers is $28.18 \mathrm{GHz}$ (i.e., $2 \mathrm{FSRs}$ ). Four representative snapshots are displayed as the detuning mismatch $\left(\delta_{1}-\delta_{2}\right)$ changes from -20 to $20 \mathrm{MHz}$. When the mismatch is close to $0 \mathrm{MHz}$, i.e., the lattice trap's group velocity matches that of solitons which are pumped primarily from the primary laser, typical DKS signatures (i.e., soliton steps) are observed, which means that the DKSs in bistability can be generated and trapped. As the mismatch increases, at first the solitons generated by the primary pump cannot survive after the secondary pump is efficiently coupled into the cavity. However, when the mismatch reaches approximately $20 \mathrm{MHz}$ [panel 4 of Fig. 1(e)], the DKS step corresponding to the secondary laser is observed to coincide within the step region of the primary soliton, indicating the appearance of multistability as two different kinds of classical bistable DKSs are excited simultaneously.

We model this scenario using the Lugiato-Lefever equation (LLE) [50-52], which we express as

$$
\begin{aligned}
\frac{\partial \tilde{A}_{\mu}(t)}{\partial t}= & \left(-\frac{\kappa}{2}+i(2 \pi \delta)+i D_{\mathrm{int}}(\mu)\right) \tilde{A}_{\mu} \\
& -i g \mathcal{F}\left[|A|^{2} A\right]_{\mu}+\left(\delta_{\mu 0}^{\prime}+\beta e^{i \Omega t} \delta_{\mu n}^{\prime}\right) \sqrt{\kappa_{\mathrm{ex}}} \cdot s_{\mathrm{in}},
\end{aligned}
$$

where $\tilde{A}_{\mu}$ and $A$ are the spectral and temporal envelopes of DKSs, respectively [related via $A(t)=\sum_{\mu} \tilde{A}_{\mu} e^{-i \mu D_{1} t}$ ], $\kappa$ is the cavity loss rate, $g$ is the single-photon-induced Kerr frequency shift, $\kappa_{\mathrm{ex}}$ is the fiber coupling rate, $\left|s_{\text {in }}\right|^{2}$ denotes the pump power, $\delta_{\mu 0}^{\prime}$ and $\delta_{\mu n}^{\prime}$ are Kronecker delta functions, $D_{\text {int }}(\mu)$ is the integrated dispersion that is defined as $D_{\text {int }}(\mu)=\omega_{\mu}-\omega_{0}-\mu D_{1}$, and $\mathcal{F}[]_{\mu}$ represents the $\mu$ th frequency component of the Fourier series. The secondary pump is incorporated into the driving term with a ratio $\beta$ and an extra detuning $\Omega$. With these notations the primary pump (i.e., the pump with the larger effective detuning) exhibits a mode number of $\mu=0$, while $n$ is the secondary pump mode number.

We perform simulations for the two representative scenarios of conventional dual-pumped soliton lattice trapping and the coexistence of distinct DKSs, respectively. The top subplot in Fig. 1(f) shows the generation and the evolution of DKSs as two lasers of identical detunings are swept across the resonances. Multiple solitons are generated after the modulation instability (MI) stage and they are attracted to certain locations by the lattice traps (which are visible in the background; see Supplemental Material [53] for more details). However, as seen in the bottom subplot, when there is a significant amount of detuning mismatch $(70 \kappa)$, as the two lasers are scanned over the resonances, both of them generate their own DKS after the MI (at locations around $200 \times 10^{3}$ and $900 \times 10^{3}$ round-trip counts) and the two different DKS states coexist, without being affected by the rapidly drifting lattice traps that are constituted by the background continuous wave (cw) drive fields.

\section{COMPOSITE SOLITONS}

We first investigate the case where two pump lasers are closely spaced (i.e., $|n| \leq 3$ ). Two telecom band lasers with a linewidth of $\sim 10 \mathrm{kHz}$ are operated in free-running conditions, pumping two resonances after amplification. Figure 2(a) presents an optical spectrum of the coexisting DKSs for the secondary laser pumping the adjacent mode (i.e., $\mu=-1$.) The DKS examination setup is similar to the one in Ref. [41], which uses an electrical spectrum analyzer (ESA) to monitor the DKS repetition rate $f_{\text {rep }}$ and a vector 
(a)

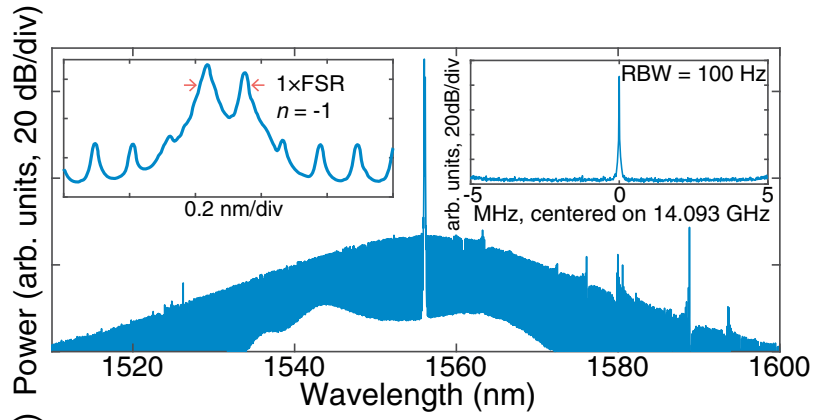

(c)

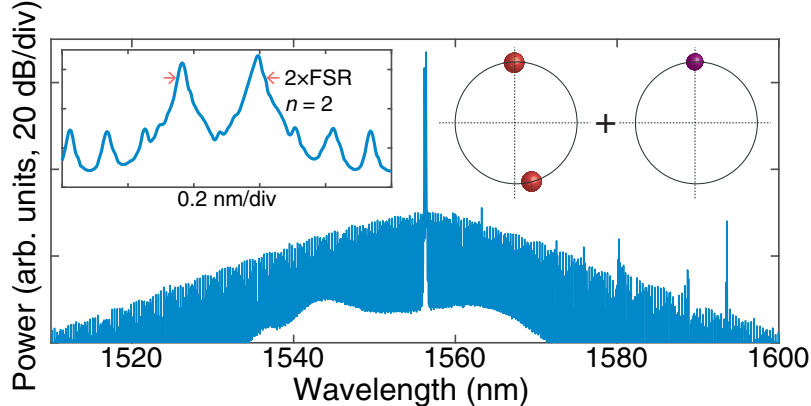

(b)

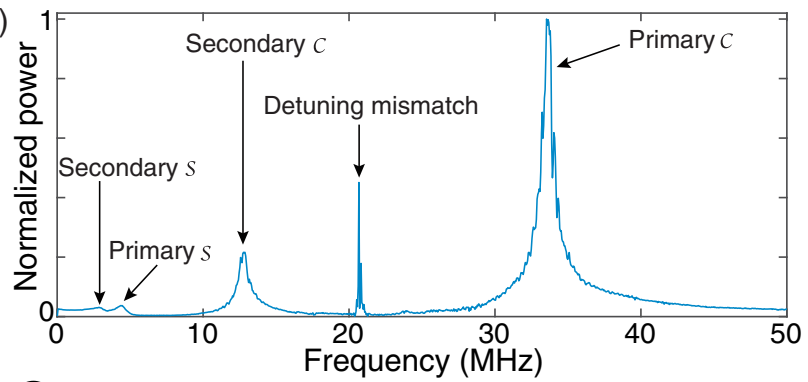

(d)

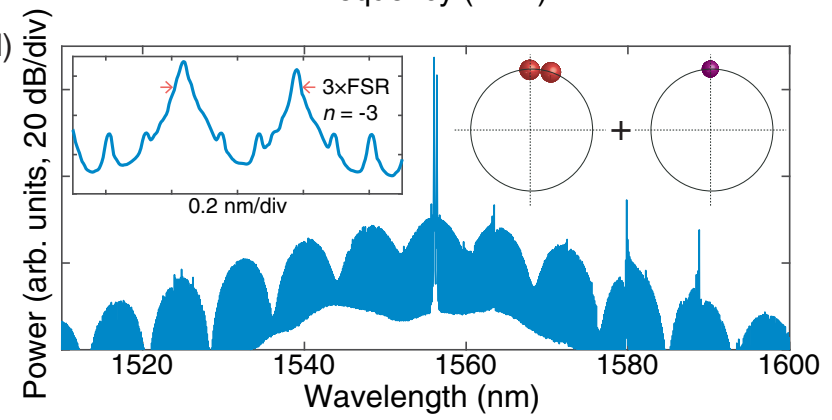

FIG. 2. Observation of dual-pumped composite solitons. (a) Optical spectrum of a composite soliton with $n=-1$. The inset on the left shows the central part of the spectrum. The inset on the right shows the measured single repetition rate of the composite solitons. The resolution bandwidth (RBW) of the measurement is $100 \mathrm{~Hz}$. (b) The measured response of pump probing by vector network analyzer. Two pairs of "S resonance" and " $\mathcal{C}$ resonance" are indicated. (c),(d) Two examples of generated composite soliton spectra corresponding to the pumping schemes shown in the left-hand insets with $n=2$ and $n=-3$, respectively. The qualitative illustrations of constituent primary and secondary soliton patterns are exhibited in the right-hand insets, respectively.

network analyzer (VNA) to measure the transfer function of the Kerr resonator [66-68]. The optical spectrum shows that a primary DKS and a secondary DKS coexist, and the transfer function in Fig. 2(b) also shows two pairs of typical "S resonance" and " $\mathcal{C}$ resonance," each of which corresponds to a unique DKS state. Since the second-order dispersion coefficient $\left(\left(D_{2} / 2 \pi\right)\right)$ is $2 \mathrm{kHz}$, the difference between the intrinsic repetition rates of the two solitons is equally expected to be $2 \mathrm{kHz}$. However, we observe only a single repetition rate, which suggests that the two distinct solitons form a bound state and travel with identical group velocity.

We generate multiple spectra of superpositions of primary and secondary DKSs with varied $|n|$, two of which are presented in Figs. 2(c) and 2(d) for examples. The compositions of constituent DKSs are inferred from the interference patterns [41] of the comb spectra and qualitatively shown in the insets. Despite the coexistence of multiple soliton states giving rise to a kaleidoscope of complex DKS microcomb patterns, there is always only a single repetition rate observed, which suggests that the primary solitons and the secondary solitons are stably bound together when their difference in intrinsic soliton group velocity is relatively small.

Our theoretical analysis based on coupled LLEs reveals that unlike the binding between similar DKSs in a monochromatically pumped multisoliton bound state that locks the relative phases between solitons $[29,30]$, the formation of composite solitons as studied here relies on the soliton-imposed refractive index barriers which are induced by the cross-phase-modulation (XPM) effect (seethe Appendix A and Supplemental Material [53] for details). In contrast to the large intersoliton separations in the monochromatically pumped multisoliton bound state, the separations between distinct DKSs in the case of multistability are close to the soliton durations. To verify the structures of the composite solitons, we use an electro-optic comb (EOC) to conduct ultrafast sampling [27,41]. Figure 3(a) depicts the concept of the sampling technique that allows us to measure the intersoliton separations between distinct DKSs with subpicosecond resolution (see the figure caption and Appendix B for details). Figures 3(b)-3(d) show the optical spectra and the sampled interferograms of three different composite solitons (left-hand panels) and the examples of corresponding sampled structures (right-hand panels), with averaged intersoliton separations indicated. The simulated composite soliton profiles are presented in the insets, showing remarkable agreement between the theoretically predicated intersoliton separations and the experimental results. All the measured separations are of only a few hundred femtoseconds, evidencing a direct soliton interaction that is fundamentally different from the dispersive-wave-mediated long-range interactions [29-31] between similar solitons.

\section{SOLITON COLLISIONS}

Our observation of composite solitons reveals the role of XPM in binding distinct DKSs. Naturally, one would 
(a)

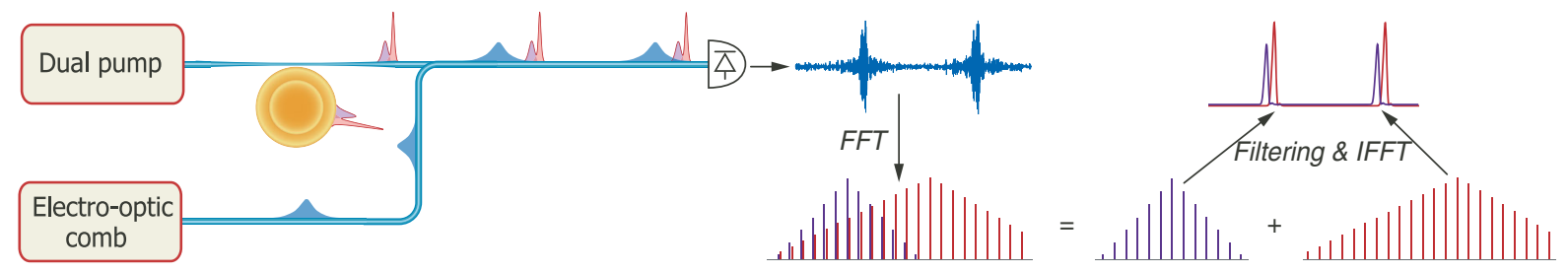

(b)
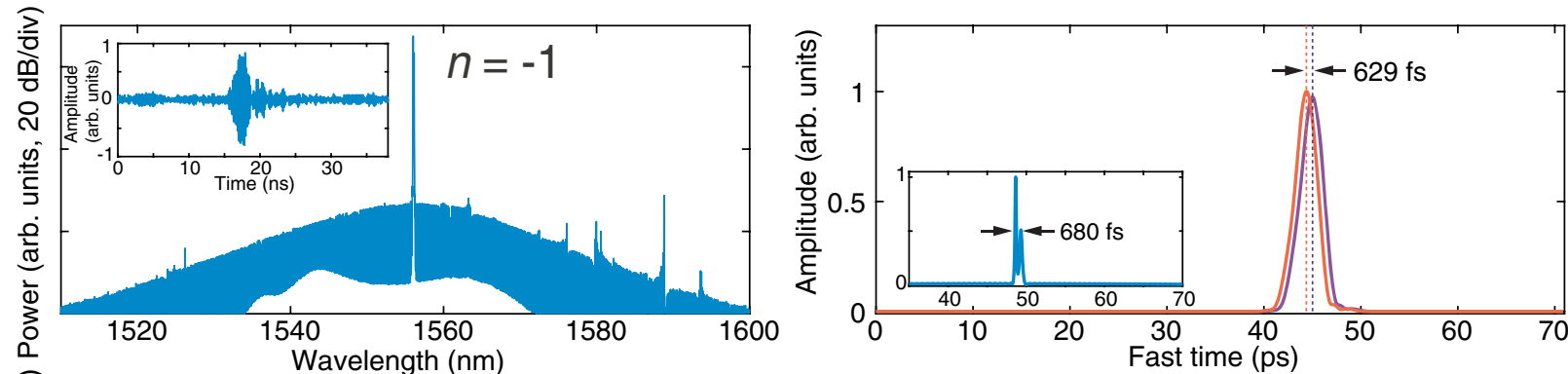

(c)
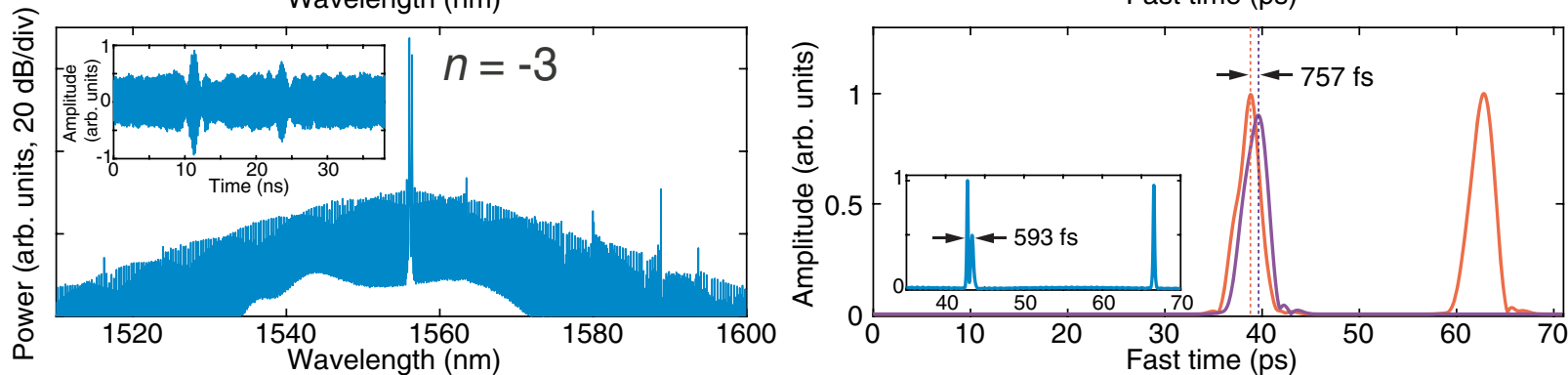

(d)
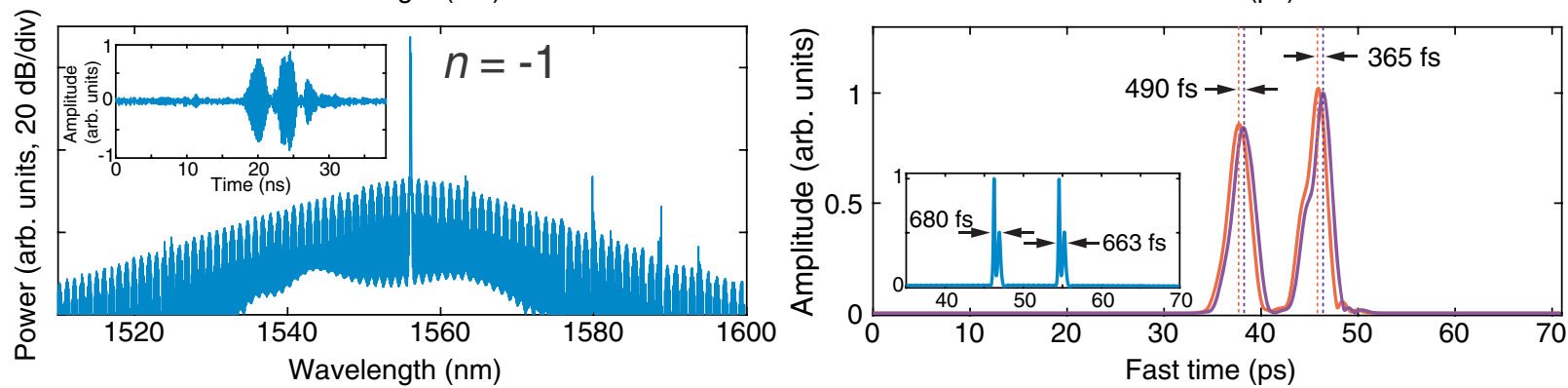

FIG. 3. Measurement of intersoliton separations. (a) Conceptual illustration of the process of determining intersoliton separations in composite solitons. Similar to the principle of the dual-comb spectroscopy, the EOC beats with the DKS at the receiving photodetector, generating interferograms [27]. Owing to the frequency offset between the primary and the secondary combs, the interferograms can be separated into the primary ones and the secondary ones, which allow the separations between distinct DKSs to be measured with a resolution that is much shorter than the temporal duration of the probing EOC pulses (see Appendix B for details). (b)-(d) The optical spectra of three composite solitons (left-hand panels) and the corresponding sampled structures (right-hand panels). The insets in the left-hand panels are interferograms sampled by the electro-optic comb. In the insets in the right-hand panels are the simulated temporal profiles. The measured and the simulated intersoliton separations are indicated.

expect that there is a limit in the inherent soliton group velocity mismatch beyond which the XPM-induced refractive index barriers cannot keep distinct solitons bound anymore. In this situation, it is not immediately obvious whether the solitons would annihilate upon collision or stably coexist with periodic collisions (thus forming a collisional soliton state). To test this regime of colliding DKSs, we increase the mode number difference $(|n|)$ between the driven resonances, leading to an intrinsic DKS repetition rate mismatch which can be approximated by $\Delta f_{\text {rep }}=|n| \times D_{2}$.
Figure 4 presents our observation of the coexistence of a primary DKS and a secondary DKS without being bound together, with $|n|=47$. Two repetition rates are observed, with a difference $\Delta f_{\text {rep }}$ of $96 \mathrm{kHz}$. Given that in our case, $D_{2}$ is $\sim 2 \mathrm{kHz}$, the measured $\Delta f_{\text {rep }}$ is in excellent agreement with the expected value. The outcoupled solitons are registered by a photodetector, and the detected power fluctuations at low frequencies $(0-7.5 \mathrm{MHz})$ are presented in Fig. 4(c). We observe multiple spectral peaks at $\Delta f_{\text {rep }}$ and its harmonics. We also simulate the collisional soliton state with the single-LLE model to calculate the spectrum 

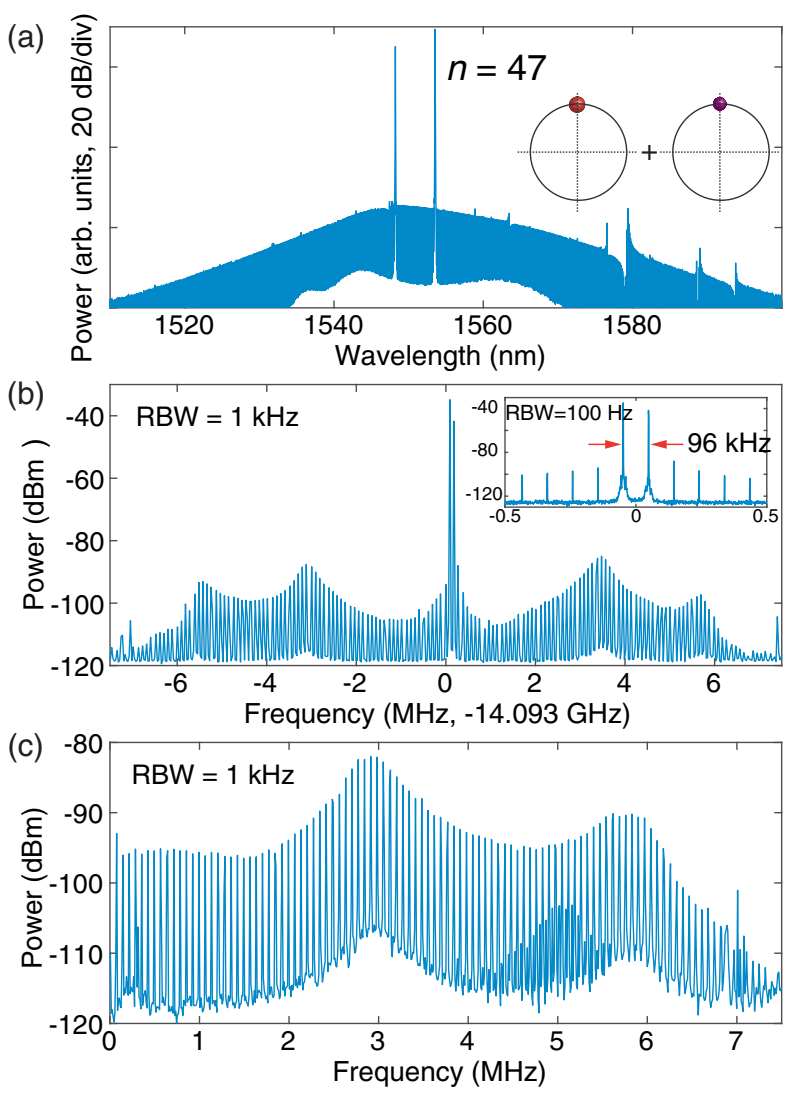

FIG. 4. Observation of soliton collisions. (a) Optical spectrum of the coexistence of a primary soliton and a secondary soliton. (b) The spectrum of the repetition rates of solitons. The inset shows the two prominent spectral peaks with a frequency difference of $96 \mathrm{kHz}$. There are also multiple sidebands with equal frequency separations that are generated by the collisions. (c) The spectrum of the output soliton power at low-frequency ranges. The overall shape resembles that of the sidebands around the repetition rates, having two peaks around 3 and $5.7 \mathrm{MHz}$ which we attribute to the peaks around the $\mathcal{S}$-resonance frequencies in the noise transfer function [67] in a modulated DKS microcomb.

of the power fluctuations of DKSs, showing excellent agreement with the experimental results (see Appendix C for details).

We use the EOC-assisted sampling technique to image the periodic soliton collisions. Figure 5(a) shows the comb spectra and the VNA traces of two collisional states with $n=29$. In Fig. 5(b) the reconstructed images of the primary DKS and the secondary DKS are shown in the upper panels and the lower panels, respectively. The red dashed lines indicate the locations where collisions occur. In Fig. 5(c) are the enlarged soliton evolutions around the collision locations that are indicated by the white dashed boxes in Fig. 5(b). We plot the fluctuations of the peak amplitudes of the imaged solitons in Fig. 5(d). The results show that upon collision DKSs exhibit strong oscillations that decay in a few microseconds, a timescale that is in agreement with the cavity photon decay time. However, the oscillation frequencies are found to be different for the primary DKSs and the secondary ones [see the difference between the red traces and blue traces in Fig. 5(d)]. While the primary DKS oscillates at around $5 \mathrm{MHz}$, the secondary DKS shows an oscillation frequency close to $3 \mathrm{MHz}$. We point out that, interestingly, the two oscillation frequencies quantitatively fit the frequencies of the $\mathcal{S}$ resonances corresponding to the primary and the secondary DKS [see the VNA spectra in Fig. 5(a)]. This observation highlights the fact that the $\mathcal{S}$ resonance is an eigenfrequency of the DKS response to modulations and perturbations, and therefore also applies to the collisional dynamics. It also shows how the DKS dynamics markedly deviates from a particlelike behavior.

\section{SWITCHING BETWEEN COMPOSITE SOLITON AND SOLITON COLLISION}

It is evident from our studies so far that, depending on the intrinsic group velocity mismatch, the coexistence of distinct solitons leads either to a bound state of composite soliton or to successive collisions. A reasonable assumption is that there exists a region of group velocity mismatch over which the two states can be switched in between each other. Here we exploit the detuning dependence of the repetition rate [69-71] to selectively tune one of the constituent solitons in order to observe the reversible switching between the composite soliton state and the state of soliton collision.

The experimental setup is schematically depicted in Fig. 6(a). With a secondary pump order $n=11$ we generate the coexistence of a single primary DKS and a single secondary DKS [see the microcomb spectrum in Fig. 6(a)], and then tune the primary detuning from 30 to $40 \mathrm{MHz}$ and then back to $30 \mathrm{MHz}$ [see the cavity resonances in Fig. 6(b1)]. At the beginning, while the output solitons do not show power fluctuations in Fig. 6(b3), we observe only one repetition rate in Fig. 6(b2), indicating that the primary soliton and the secondary soliton are bound together as a composite soliton. As the primary detuning is increased, the measured repetition rate slowly decreases due to the intrinsic repetition rate change of the primary soliton, until the single repetition rate suddenly splits into two prominent signals at a primary detuning of $\sim 34 \mathrm{MHz}$. Concurrently, the power fluctuation spectrum exhibits multiple spectral components at the frequencies which correspond to the repetition rate difference and its harmonics, indicating the dissociation of the bound state and the switching to the collisional soliton state. As the primary detuning is further increased, the higher repetition rate (which corresponds to the single secondary soliton) stays nearly the same, while the repetition rate of the primary soliton shows an overall tendency to decrease. After the primary detuning of $40 \mathrm{MHz}$ is reached, the detuning is reversed until it reaches the original $30 \mathrm{MHz}$. In doing so, the soliton group 
(a) $\stackrel{2}{=}$

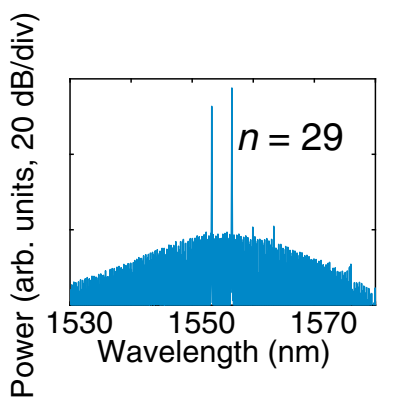

(b)
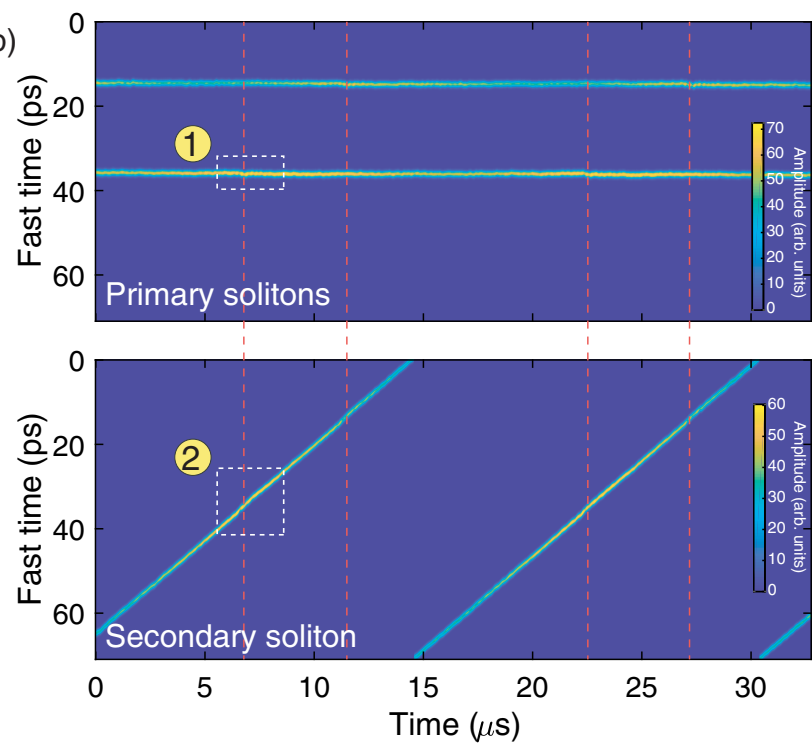

(c)

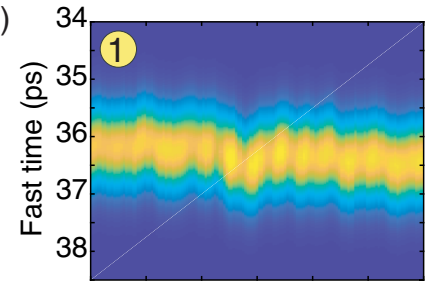

(d)

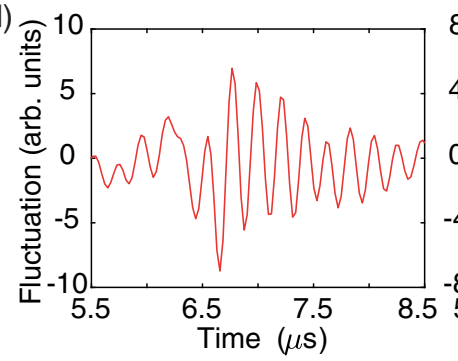

$\frac{\widehat{m}}{5}$

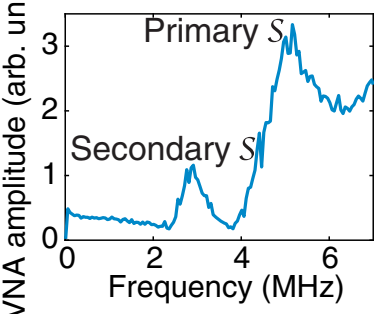

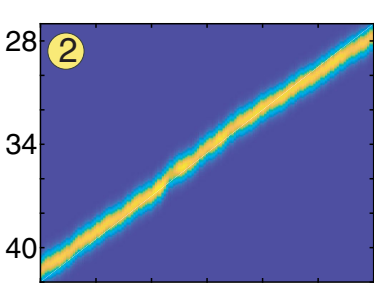

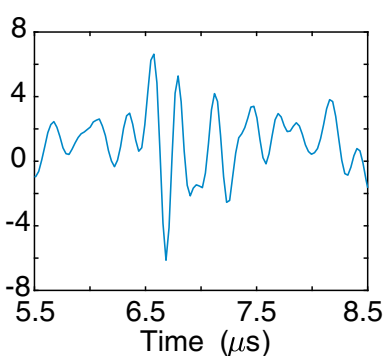

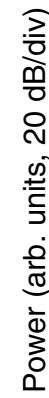
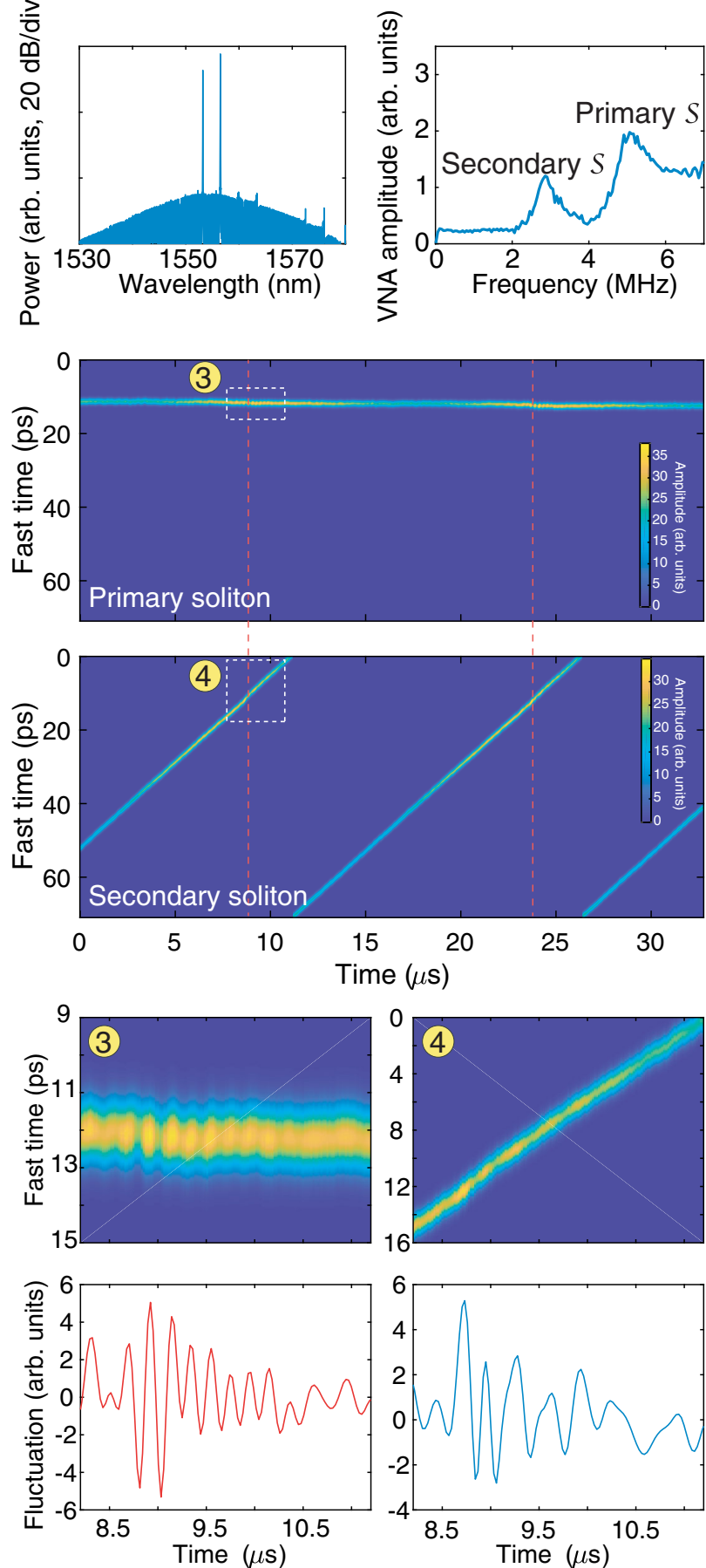

FIG. 5. Imaging of soliton collision dynamics. (a) Optical spectra and VNA response spectra of soliton collision between dual primary solitons and a single secondary soliton (left-hand panel), and between single primary soliton and single secondary soliton (right-hand panel). (b) Images of primary solitons (upper) and secondary solitons (lower) during the soliton collisions. The locations where collisions happen are indicated by the red dashed lines. (c) Enlarged images of soliton collisions. Each corresponds to the numbered white dashed box in (b). (d) The fluctuation of the peak intensities of imaged solitons when collisions occur. The primary soliton and secondary soliton show very different characteristic oscillation frequencies.

velocity mismatch is reduced, and we observe the reversal to the composite soliton state when the two repetition rates join into one. This is accompanied with the disappearance of the soliton power fluctuations at low frequencies.

\section{DISCUSSION AND CONCLUSION}

This work reported on the combined theoretical and experimental study of the Kerr multistability that allows for the generation of composite solitons, and studied in 


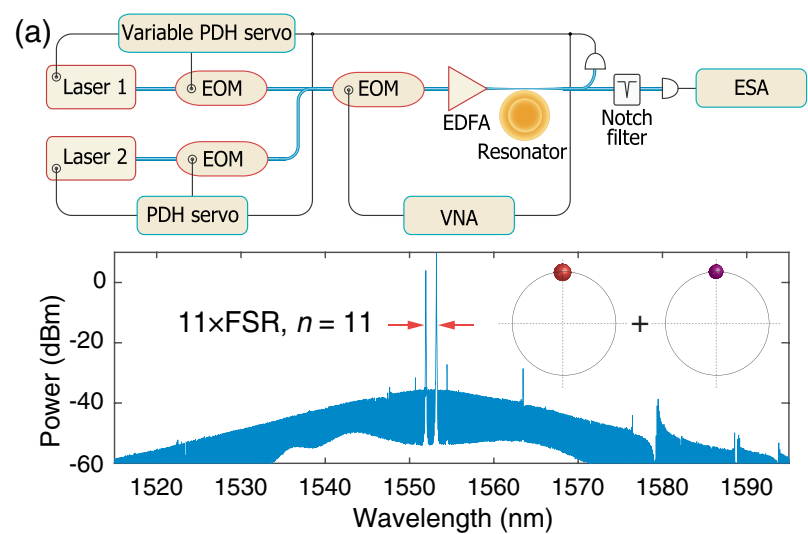

(6)
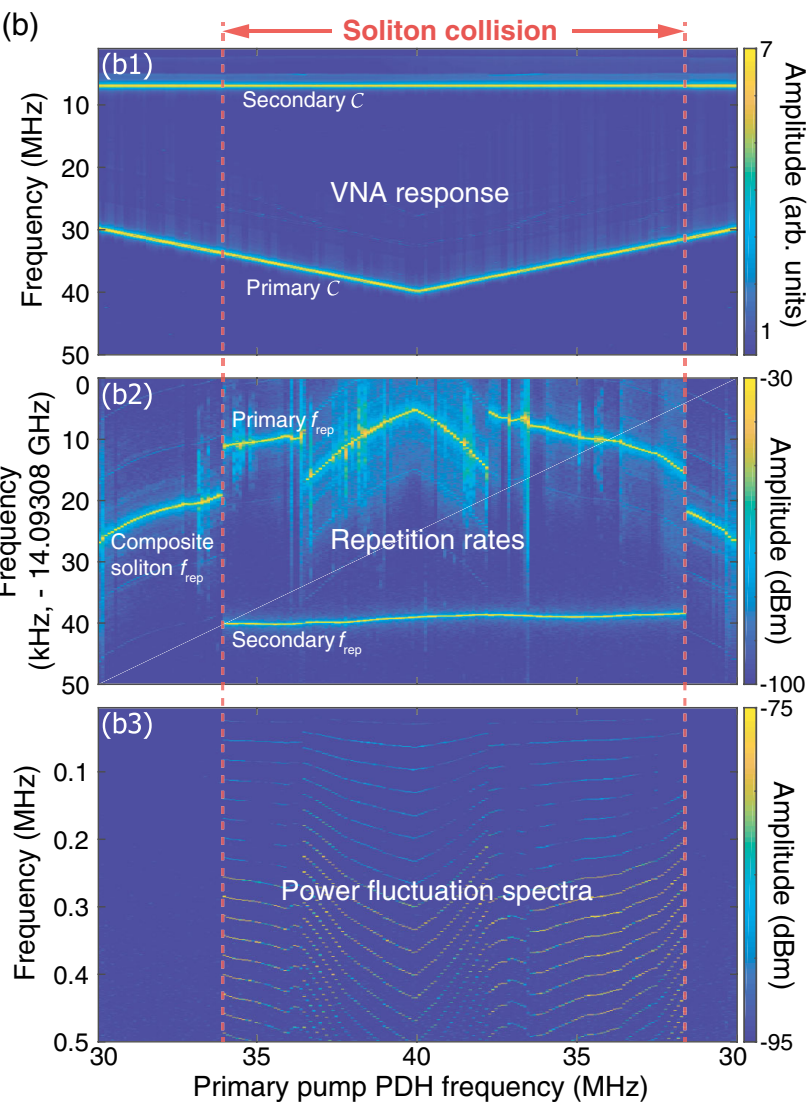

FIG. 6. Reversible switching between composite soliton state and collisional soliton state. (a) In the experimental setup (top), the two lasers are separated from each other by $\sim 11$ FSRs, and the two pump-resonance detunings are actively locked by locking the phase-modulated sidebands of lasers to the Pound-Drever-Hall (PDH) error signals. While the secondary pump-resonance detuning is unchanged, the detuning of the primary pump is varied by continuously tuning the PDH modulation frequency. The optical spectrum of the coexistence of a primary soliton and a secondary soliton (bottom). (b) The evolutions of the VNA response (b1), the repetition rate spectra (b2), and the power fluctuation spectra (b3) as the primary pump-resonance detuning is varied from 30 to $40 \mathrm{MHz}$ and then back to $30 \mathrm{MHz}$. The jumps of the primary $f_{\text {rep }}$ around $37 \mathrm{MHz}$ are attributed to a strong single dispersive wave [11] that we confirm by comparing the acquired microcomb spectra before and after the jump. The range where the state of soliton collision is switched on is denoted by the red dashed lines. particular the regime of the collisional soliton state. To the best of our knowledge, this is the first time the multistability is investigated in a bichromatic pumping setting, where two novel DKS dynamical states emerge, with the framework of bistability breaking down. In addition, the multistability generation scheme can be extended to even more complicated scenarios (e.g., using more than two lasers to pump multiple resonances with different effective detunings respectively [53]), showing potential for studying more complex soliton dynamics.

Our work casts light on several intriguing questions. Firstly, where is the boundary between the conventional bistability and the multistability in a dual-pumped resonator? Previous works $[44,72,73]$ treat the dual-pumping scheme as an approach to manipulate DKSs in the context of bistability. However, here we show that as the pumpresonance detuning mismatch increases, the multistability emerges and both pumps can support their own solitons whose existence is almost unaffected by the interference between the laser fields. Secondly, can DKSs driven at different resonances be bound together? Our work shows that, different from the relative phase-dependent long-range binding of similar DKSs, distinct DKSs can be closely bound with XPM-induced soliton barriers. We would also like to mention the relevance of our findings to studies of the optical analog of event horizons [74-77], which use similar index barriers to trap weak probe waves in fibers. Our work enriches the field by showing that in Kerr resonators solitons can be either blocked by or released from the rotating boundaries imposed by other DKSs, which yields potential applications in pulse timing jitter compensation [78]. Lastly, what are the eigenfrequencies involved in DKS dynamics? Earlier works $[67,68]$ suggested that the $\mathcal{S}$-resonance frequencies correspond to the so-called DKS relaxation oscillation. In this work the imaging of colliding solitons shows that the solitons exhibit excitable oscillations at the same frequencies of $\mathcal{S}$ resonance, which is closely related to fundamental soliton characteristics given that DKSs breathe at the same frequencies before they annihilate. Therefore the $\mathcal{S}$ resonance frequencies can be understood as the intrinsic eigenfrequencies of DKS dynamics that can be excited by applying small perturbations or modulation signals.

For potential applications in metrology and spectroscopy, this work develops an approach to generate microcombs with interlocked repetition rates. One should note that for most of the experiments the two pumping lasers are in free-running condition, showing the remarkable robustness of the interlocking mechanism. In addition, the DKS collisional state can be related to earlier studies on soliton-based wavelength-division multiplexing fiber telecommunication systems, in which fast and numerous collisions between solitons of dissimilar wavelengths (and hence different group velocities) can reduce the detrimental effects caused by nonlinear interactions 
between solitons [79-82]. In this regard, microresonator DKSs may provide a compact and convenient platform to study soliton nonlinear interactions that would require massive length of fiber or waveguide in conservative systems. Moreover, our study on the soliton collision is of importance for dual-comb spectroscopy based on the spatially multiplexed DKSs as the sidebands introduced by the intermodulation between colliding solitons manifest themselves as artifacts in the dual-comb interferograms [25].

The code and data used to produce the plots within this article are available by following the link in Ref. [83].

\section{ACKNOWLEDGMENTS}

W. W. thanks Hairun Guo for helpful discussions on the simulation. The authors thank M. H. Anderson for experimental assistance and J. C. Skehan for proofreading the manuscript. This publication was supported by Contract No. D18AC00032 (DRINQS) from the Defense Advanced Research Projects Agency (DARPA), Defense Sciences Office (DSO), and funding from the Air Force Office of Scientific Research, Air Force Material Command, USAF under Grant No. FA9550-19-1-0250.

\section{APPENDIX A: COUPLED LUGIATO-LEFEVER EQUATIONS}

With the assumption that XPM is the dominant mechanism that enables the binding of distinct solitons, we use a set of coupled LLEs to describe the intracavity fields that are driven by the primary pump and the secondary pump, respectively. Since the two fields have different carrier frequencies, we simplify the situation by ignoring all the phase-related coupling terms and consider only the XPM between the two fields. In the frequency domain, the two soliton states correspond to two sets of optical frequency combs, and here for clarity we denote them as the primary $(\mathcal{P})$ comb and the secondary $(\mathcal{S})$ comb, respectively. In a frame that is comoving with the waveform in the primary soliton (free spectral range $D_{1}^{(\mathcal{P})} / 2 \pi$ ) centered at the pump frequency $\left(\omega_{p}\right)$, the coupled equations are written as

$$
\begin{aligned}
\frac{\partial \tilde{A}_{\mu}^{(\mathcal{P})}(t)}{\partial t}= & \delta_{\mu 0}^{\prime} \sqrt{\kappa_{\mathrm{ex}}} s_{\mathrm{in}}+\left(-\frac{\kappa}{2}+i(2 \pi \delta)+i D_{\mathrm{int}}(\mu)\right) \tilde{A}_{\mu}^{(\mathcal{P})} \\
& -i g\left(\mathcal{F}\left[\left|A^{(\mathcal{P})}\right|^{2} A^{(\mathcal{P})}\right]_{\mu}+\mathcal{F}\left[2\left|A^{(\mathcal{S})}\right|^{2} A^{(\mathcal{P})}\right]_{\mu}\right),
\end{aligned}
$$

$$
\begin{aligned}
\frac{\partial \tilde{A}_{\mu}^{(\mathcal{S})}(t)}{\partial t}= & \delta_{\mu n}^{\prime} \beta \sqrt{\kappa_{\mathrm{ex}}} s_{\mathrm{in}} \\
& +\left(-\frac{\kappa}{2}+i(2 \pi \delta-\Omega)+i D_{\mathrm{int}}(\mu)\right) \tilde{A}_{\mu}^{(\mathcal{S})} \\
& -i g\left(\mathcal{F}\left[\left|A^{(\mathcal{S})}\right|^{2} A^{(\mathcal{S})}\right]_{\mu}+\mathcal{F}\left[2\left|A^{(\mathcal{P})}\right|{ }^{2} A^{(\mathcal{S})}\right]_{\mu}\right),
\end{aligned}
$$

where $\tilde{A}_{\mu}^{(\mathcal{P})}$ and $\tilde{A}_{\mu}^{(\mathcal{S})}$ represent the spectral field envelope of the primary and the secondary combs, respectively, and $A^{(\mathcal{P})}$ and $A^{(\mathcal{S})}$ are the temporal amplitudes of the intracavity field. The last terms in the equations are the XPM-induced coupling terms [2]. In the simulation, we neglect the higherorder dispersion to reasonably simplify the analysis. As a result, the comoving frames for the two fields are different only due to the effect of the second-order dispersion, which leads to $D_{1}^{(\mathcal{P})}=D_{1}^{(\mathcal{S})}+n D_{2}$. Here, $\left(D_{1}^{(\mathcal{P})} / 2 \pi\right)$ and $\left(D_{1}^{(\mathcal{S})} / 2 \pi\right)$ are the FSRs at the primary and the secondary pumped resonances, respectively.

In Figs. 7(a) and 7(b), we show the evolution of the intracavity fields that are simulated by the coupled LLEs. For the simulation we set $\mu=-5, \delta=(150 \kappa / 2 \pi)$, and $\Omega=(100 \kappa / 2 \pi)$. The two distinct DKSs are seeded at wellseparated locations at the beginning of the simulation. Since the secondary soliton has a repetition rate that is lower than that of the primary soliton by $10 \mathrm{kHz}$, it meets the primary soliton at round-trip counts of $\sim 200 \times 10^{3}$. Then the two solitons start to move together, showing that the composite soliton is formed. To compare the simulation results with the single-LLE model, we also run the simulation based on Eq. (1). In Fig. 7(c) the result is presented, showing excellent agreement with those derived with Eq. (A1). The amplitudes of the intracavity fields at the end of the simulations are plotted in Fig. 7(d). The comparison shows that the coupled LLEs yield an intersoliton separation that is slightly larger than the result based on the single-LLE model, which we attribute to the simplifications made to the coupled LLEs. Despite this minor discrepancy, the coupled LLEs confirm that the formation of the composite soliton is mainly induced by the XPM effect and that the constituent DKSs interact with each other in a short range that is of the order of the DKS durations. With a seminumerical method we further analyze the binding mechanism due to the XPM effect. Details can be found in the Supplemental Material [53].

In addition to the simulations based on the coupled-LLE model, we also introduce strong dispersive waves in the single-LLE model by imposing large frequency deviations on some particular modes. We use the model to extensively simulate the evolution of multiple solitons in the bichromatically driven multistability. The results reveal that while the similar solitons can form bound states due to dispersive waves, the binding of distinct solitons is only possible when they are in close proximity. Such results are expected because the dispersive-wave-mediated binding relies on a fixed relative phase between solitons, but the relative phase is never fixed for distinct solitons. To further corroborate the XPM-induced binding analysis, in Supplemental Material we directly add index barriers of different amplitude in the single-LLE model to study the motion of solitons [53]. Our further investigation shows that under the influence of an intensive index barrier such as the one created by a dissimilar intracavity soliton via XPM, solitons 

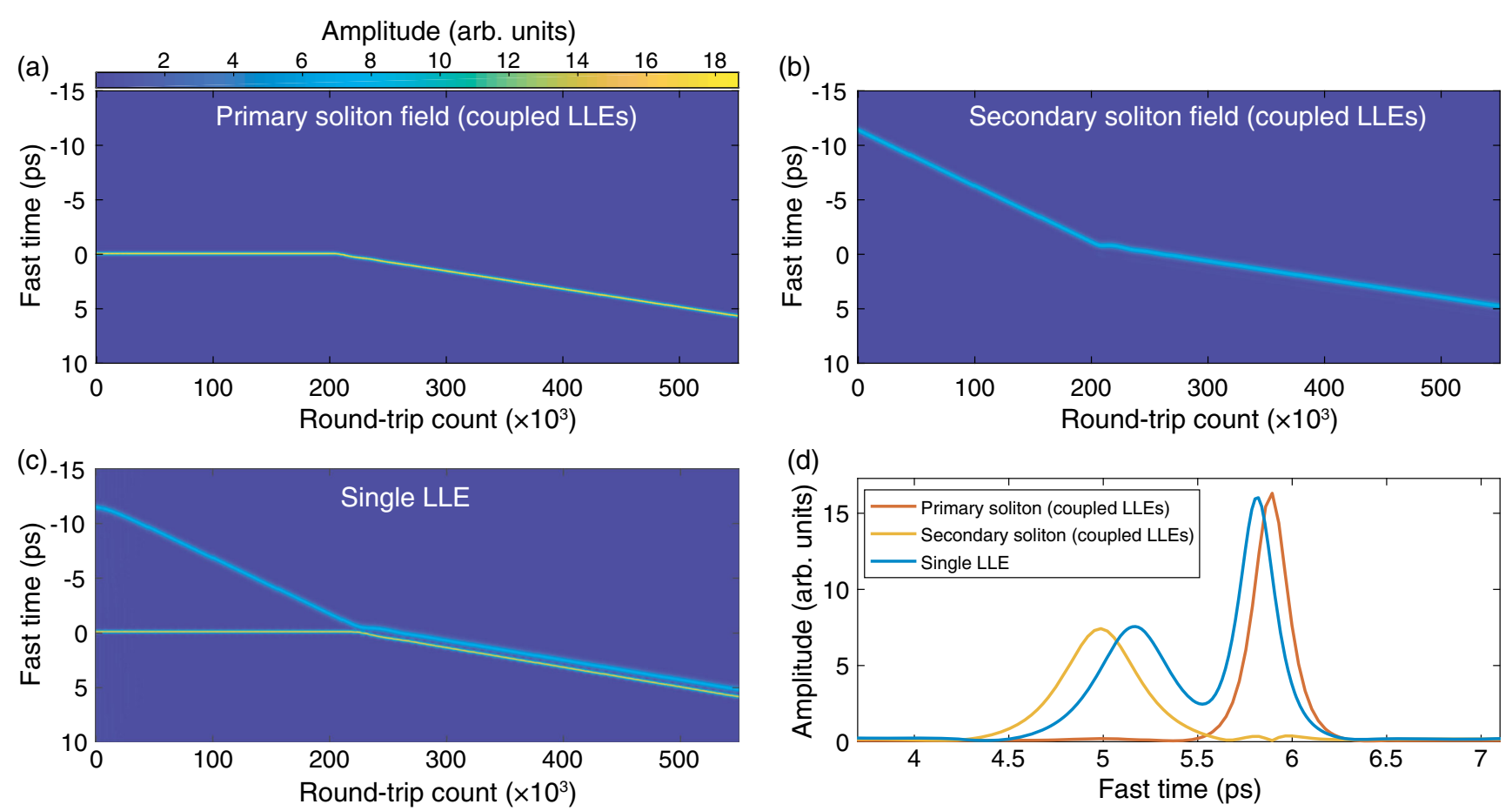

FIG. 7. Comparison between the single-LLE model and the model of the coupled LLEs. (a),(b) The respective evolutions of the primary field and the secondary field calculated by the coupled LLEs show the binding of a primary soliton and a secondary soliton. (c) The same binding process simulated with the single-LLE model. (d) The final frames in each simulated field evolution.

can be blocked out of an index barrier with repulsive effect. Such repulsive effect induced by a positive index change in anomalous dispersion is counterintuitive and can be attributed to the unique DKS feature of the coexistence of DKS and $\mathrm{CW}$ background.

\section{APPENDIX B: DETAILS OF ELECTRO-OPTIC SAMPLING}

As depicted in Fig. 8(a), the EOC is generated by modulating a 1555-nm CW laser with a Mach-Zehnder modulator (MZM) and an array of electro-optic modulators (EOMs) [27]. This modulation frequency sets the sampling rate to be $\sim 30-40 \mathrm{MHz}$. The carrier frequency of the EOC is adjusted such that the beat frequencies between the electro-optic comb and the composite solitons are in the range of $4.5-6.5 \mathrm{GHz}$, which can be properly bandpass filtered. After being amplified by an EDFA, the optical spectrum of the EOC is displayed in Fig. 8(b). The more intensive spectral components at the longer wavelengths are caused by the unbalanced gain of the EDFA for different spectral ranges. Figure 8(c) shows the radiofrequency (rf) spectrum of the interferograms corresponding to the data in Fig. 3(b). The subsets of the spectral components that belong to the primary DKS comb and the secondary DKS comb are separated, as indicated by the different colors. After applying the inverse fast Fourier transform (IFFT) to the primary and the secondary rf combs, respectively, we reconstruct the interferograms of the primary and the secondary DKS in the time domain [41], which are shown in Fig. 8(d). Because of the limited optical bandwidth of the EOC (and hence the large width of the probing pulses), the temporal widths of the envelopes of the interferograms are significantly larger than those of the DKS, as can be seen in Fig. 8(d) as well as in the sampled structure examples in the right-hand panels in Figs. 3(b)-3(d). For the same reason, the amplitudes of the sampled structures do not correctly reveal the relative DKS amplitudes. However, measuring the temporal locations of the maximum amplitudes of interferogram envelopes allows us to determine the intersoliton separation between the primary and the secondary DKS with a resolution that is not limited by the EOC bandwidth. Each of the measured intersoliton separations shown in Fig. 3 is averaged over $1.2 \times 10^{4}$ interferograms. In Fig. 8(e) we plot the total $1.2 \times$ $10^{4}$ data points of the measured intersoliton separations for the averaged value of 629 fs presented in Fig. 3(b). The standard deviation is calculated to be $107 \mathrm{fs}$, which is $17.1 \%$ of the averaged separation. The separation fluctuations may be introduced by the amplitude and width fluctuations of individual solitons, and can degrade the mutual coherence of the synchronized repetition rates, causing extra noise especially in short timescales.

\section{APPENDIX C: SIMULATION OF SOLITON COLLISION}

We simulate the soliton collision state with the singleLLE model expressed by Eq. (1). The secondary mode 

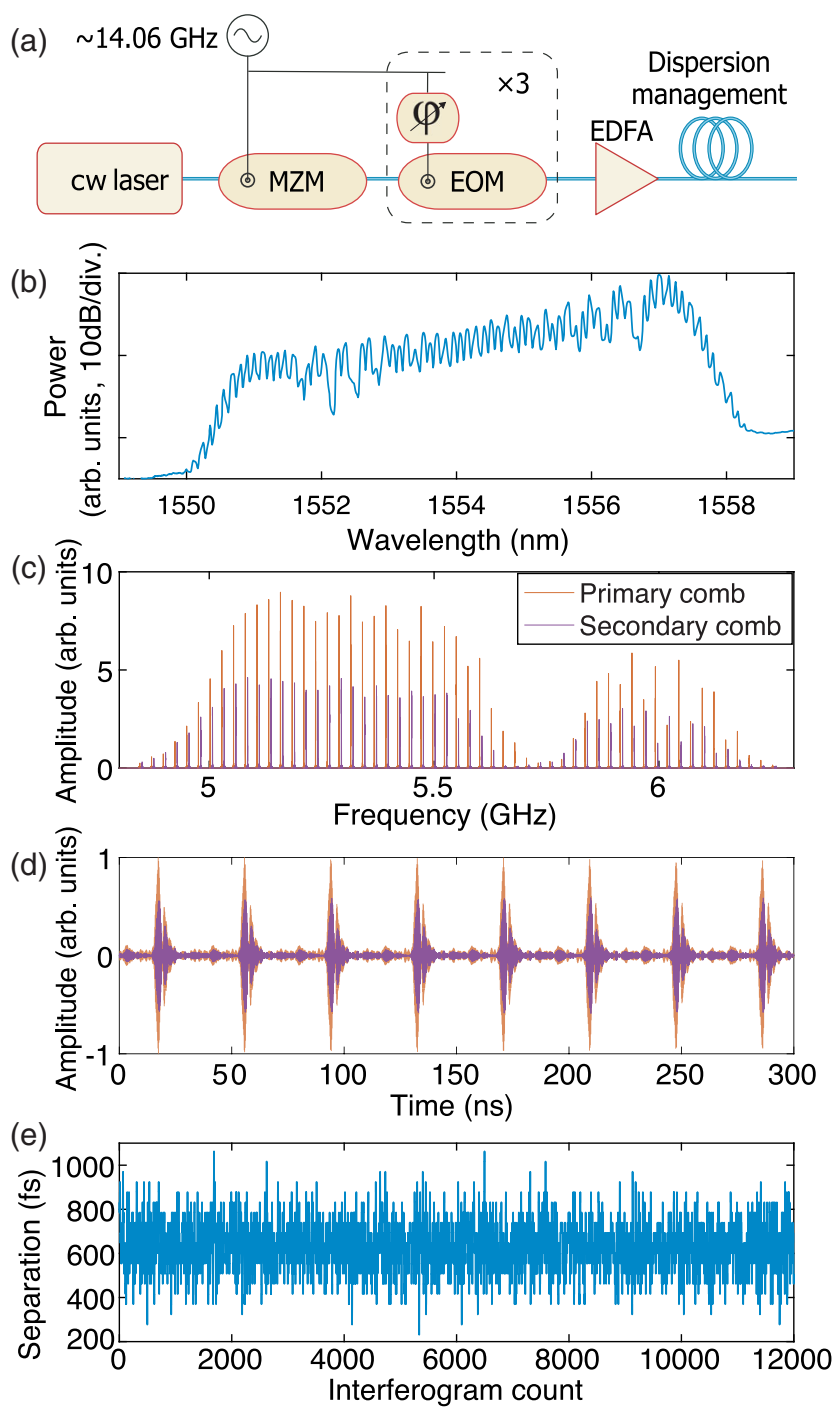

FIG. 8. Electro-optic sampling of solitons. (a) Scheme of the electro-optic comb generation. MZM: Mach-Zehnder modulator. (b) The optical spectrum of the electro-optic comb after amplification. (c) The spectra of the interferograms corresponding to the primary DKS and the secondary DKS, respectively. (d) The reconstructed interferograms with a temporal length of $300 \mathrm{~ns}$. The relative amplitudes of the interferograms are adjusted in order to clearly show both the interferogram trains. (e) The measured $1.2 \times 10^{4}$ intersoliton separations, whose averaged value of $629 \mathrm{fs}$ is shown in Fig. 3(b).

order $\mu=47$ is the same as that in the experiment shown in Fig. 4 . The primary pump-resonance detuning $(2 \pi \delta)$ is set to be $70 \kappa$, and the dual-pumping detuning mismatch $(|\Omega|)$ is $50 \kappa$. The second-order dispersion coefficient $\left(D_{2} / 2 \pi\right)=$ $2 \mathrm{kHz}$ and third-order dispersion coefficient $\left(D_{3} / 2 \pi\right)=$ $-1 \mathrm{~Hz}$ are included in the resonator dispersion. A single primary DKS and a single secondary DKS are seeded, and the whole simulation includes $2.1 \times 10^{6}$ round-trip times. Figures 9(a) and 9(b) show two collision events in the intracavity field evolution and the corresponding
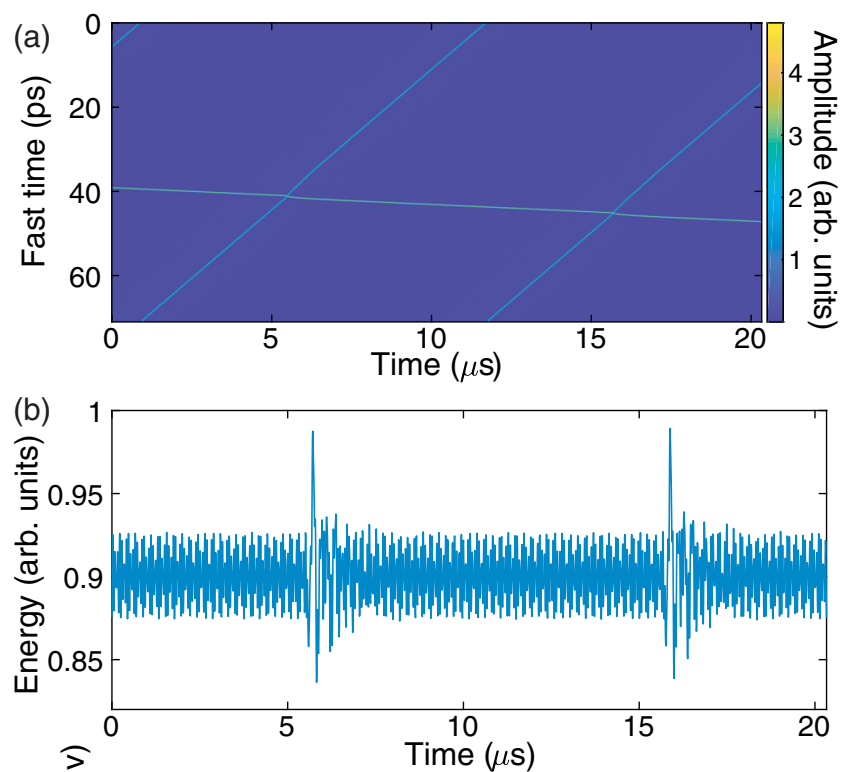

(c)

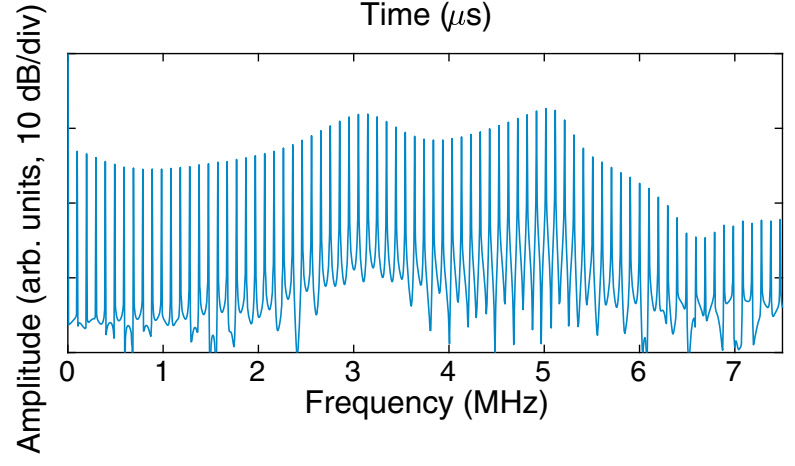

FIG. 9. Simulation of soliton collision. (a) Intracavity field amplitude evolution showing two collision events. (b) The corresponding intracavity energy evolution. (c) The calculated spectrum of the intracavity energy fluctuations.

intracavity energy fluctuations, respectively. Totally 15 soliton collision events are shown in the simulation, corresponding to a collision frequency of approximately $98 \mathrm{kHz}$. The intracavity energy fluctuations in the time domain are used to calculate the spectrum presented in Fig. 9(c), which is in excellent agreement with the experimentally measured power fluctuation spectrum shown in Fig. 4(c).

One should note that in the conventional monochromatic pumping situation the collision between two similar solitons is generally unstable because the overlap of two solitons essentially creates a high-order soliton that is forbidden in dissipative Kerr cavities. This is why the soliton collisions induced by injected potential wells result in either soliton annihilation or merging [34,35]. However, the multistability-enabled soliton collision is dynamically stable because during each collision period even the two distinct solitons overlap with each other spatial-temporally for a short time; they never overlap in the frequency domain. Therefore they cannot form high-order solitons to become dynamically unstable. 


\section{APPENDIX D: SIMULATION OF SWITCHING FROM COMPOSITE SOLITON TO SOLITON COLLISION}

The switching phenomenon reported in Fig. 6 is numerically simulated with the single-LLE model of Eq. (1). We repeated similar simulations with varied $n$ and we found that the switching happens with $|n|=10$, which is very close to the experimental value of 11 . Figure 10(a) shows the simulation result when switching from composite soliton to soliton collision happens at $n=10$. The simulation starts with a composite soliton with the primary detuning $\delta_{\mathcal{P}}=-80 \kappa$ and the secondary detuning $\delta_{\mathcal{S}}=-30 \kappa$. In order to include the effect of detuningdependent repetition rate shift due to higher-order dispersion [71], the third-order dispersion coefficient $\left(D_{3} / 2 \pi\right)=-4 \mathrm{~Hz}$ is incorporated into the LLE model. To reproduce the experimental condition, $\delta_{\mathcal{S}}$ is kept the same during the whole simulation while $\delta_{\mathcal{P}}$ is continuously decreasing. The composite soliton decomposes when $\delta_{\mathcal{P}}$ reaches $-107 \kappa$, switching to the collisional state. We numerically compute the instantaneous soliton repetition
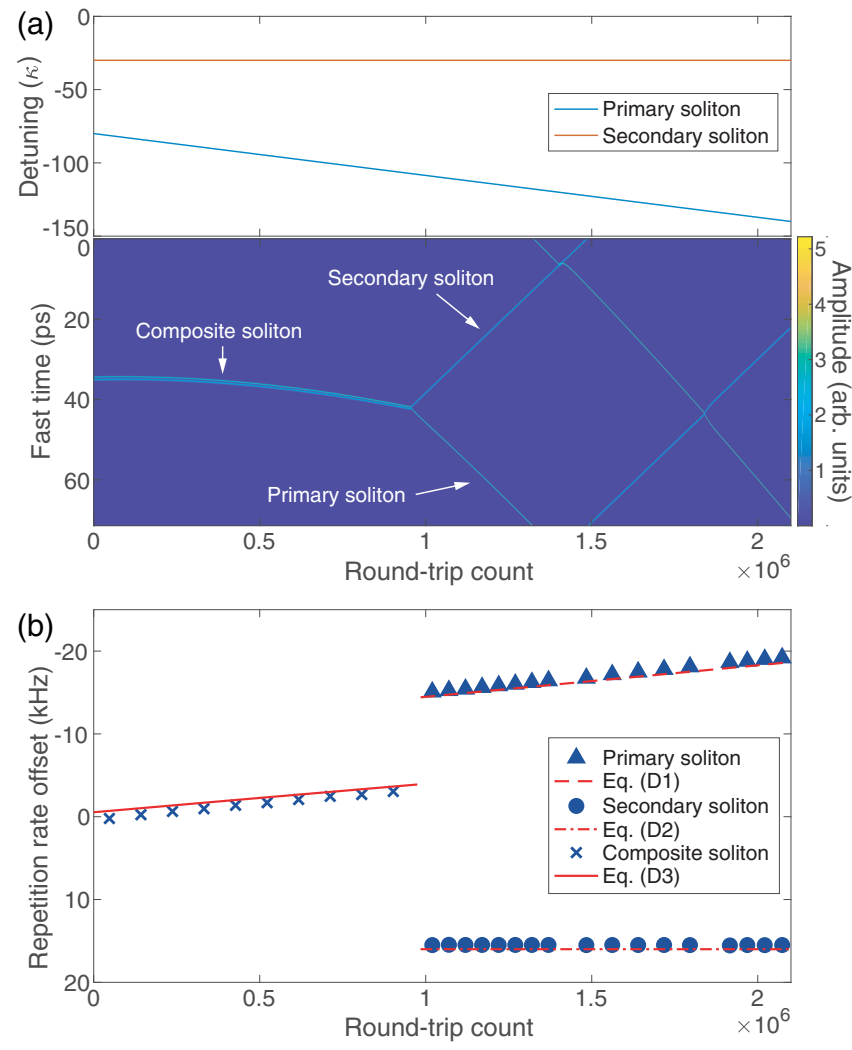

FIG. 10. Simulation of switching. (a) Evolution of the intracavity field when a composite soliton decomposes into two unbound distinct solitons. The effective detunings of the primary pump and the secondary pump are shown in the upper panel. (b) The numerically computed instantaneous soliton repetition rates relative to $\left(D_{1} / 2 \pi\right)$. The theoretically calculated repetition rates are plotted in red curves. rates (relative to $\left(D_{1} / 2 \pi\right)$ ), which are displayed in Fig. 10(b). After the switching the repetition rate mismatch is approximately $30 \mathrm{kHz}$, showing excellent agreement with the experimental observation in Fig. 6.

Analytical expression of the repetition rate shift of the primary soliton due to third-order dispersion can be found in [71]:

$$
\Delta f_{\mathcal{P}}=\frac{\delta_{\mathcal{P}} D_{3}}{3 D_{2}}
$$

And similarly, the secondary soliton repetition rate shift can be written as

$$
\Delta f_{\mathcal{S}}=\frac{n D_{2}}{2 \pi}+\frac{\delta_{\mathcal{S}} D_{3}}{3 D_{2}}
$$

Furthermore, by using the method similar to the procedure in Ref. [41] based on the conservation of soliton momentum, we derive the repetition rate shift of the composite soliton as

$$
\Delta f_{\mathcal{C}}=\frac{n D_{2} \sqrt{\delta_{\mathcal{S}}}}{2 \pi\left(\sqrt{\delta_{\mathcal{P}}}+\sqrt{\delta_{\mathcal{S}}}\right)}+\frac{D_{3}}{3 D_{2}}\left(\delta_{\mathcal{P}}+\delta_{\mathcal{S}}-\sqrt{\delta_{\mathcal{P}} \delta_{\mathcal{S}}}\right)
$$

In Fig. 10(b) we plot the repetition rate shifts based on the analytical expressions. Remarkable agreement between the theory and the numerical results is obtained.

\section{APPENDIX E: PUMPING ONE RESONANCE WITH TWO LASERS}

A special case of the bichromatic pumping is that the two lasers are used to pump the same cavity resonance. Strictly speaking, this case does not belong to the bichromatic pumping category as the beating of the two lasers does not create any intracavity potential well. Instead, modulation with a period that is orders of magnitude larger than the round-trip time is executed. We perform the simulation of this case, using the same parameters that are used in Fig. 1(f), except that the two lasers are driving the same mode resonance of $\mu=0$. The result is shown in Fig. 11(a). Since the difference between the two pumping frequencies is the pump-resonance detuning difference, which is smaller than the FSR by around 3 orders of magnitude, the coexistence of the two laser fields essentially introduces a slow modulation in the intracavity field. One period of the slow modulation takes around 1000 round-trip times; therefore, the modulation is not visible in the background field in Fig. 11. In addition, in this simulation where highorder dispersion and Raman effect are not considered, the primary and the secondary solitons have the same group velocity since they are excited in the same resonance. In reality, however, because high-order dispersion almost always exists, solitons with different pump-resonance detunings exhibit dissimilar group velocities, which 

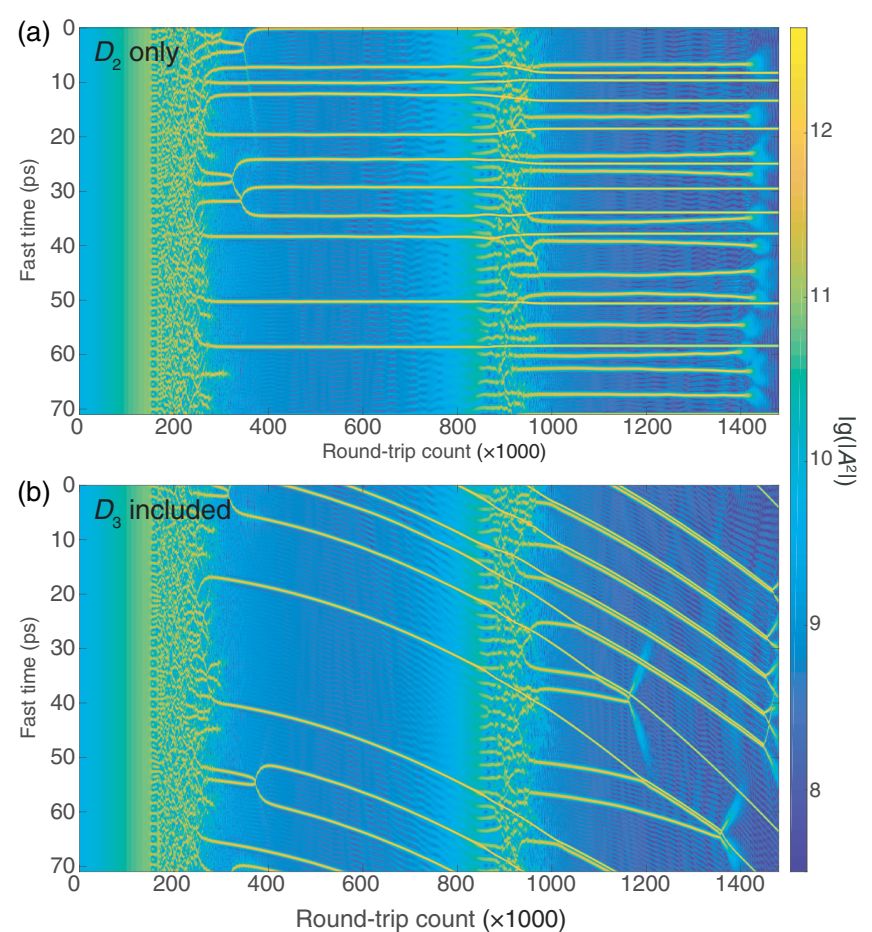

FIG. 11. Simulated intracavity intensity evolutions with two lasers driving one cavity resonance. (a) Only second-order dispersion $\left(D_{2}\right)$ is considered in the simulation. (b) Third-order dispersion $\left(D_{3}\right)$ is also included in the model. All the parameters used in the simulations are the same as those used for obtaining the results shown in the lower panel in Fig. 1(f), except that both lasers are driving the same cavity resonance of $\mu=0$.

inevitably leads to the binding of dissimilar solitons. Figure 11(b) presents the simulation result when a small amount of third-order dispersion $\left(\left(D_{3} / 2 \pi\right)=-4 \mathrm{~Hz}\right)$ is included in the model. Third-order dispersion induces detuning-dependent soliton group velocity shift [71], thus creating the group velocity mismatch between the primary and the secondary solitons. Consequently, soliton binding with subpicosecond intersoliton separations is enabled even when the two soliton species are pumped at the same resonance.

Experimentally, such single-resonance excitation with dual pumping can be realized either by carefully tuning the frequencies of the two lasers or by using a modulator such as an EOM to create the secondary pumping field from the primary laser. In Fig. 12(a) the two configurations are illustrated. One should note that when the EOM is used, the detuning mismatch is set by the modulation frequency, and only the modulation sideband with the higher frequency is used as the secondary pump (assuming that the sideband has an intensity that is lower than that of the carrier). Being far detuned from the resonance, the sideband with the lower frequency has negligible impact. Figures 12(b) and 12(c) show two multistability-enabled microcomb spectra that we use the first and the second configuration to generate, respectively. In both cases only one soliton repetition rate is (a)

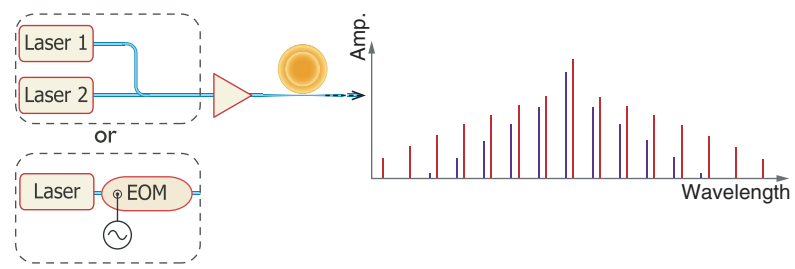

(b)

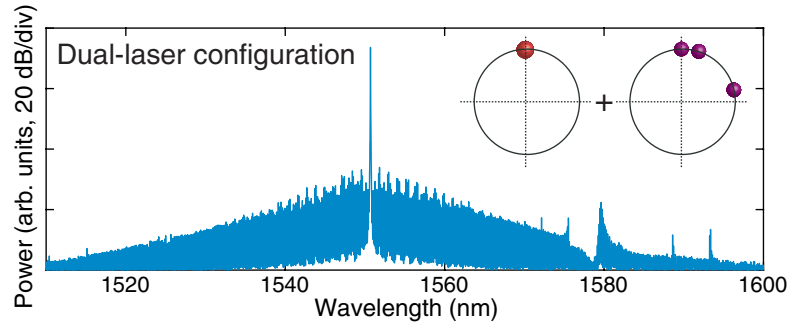

(c)

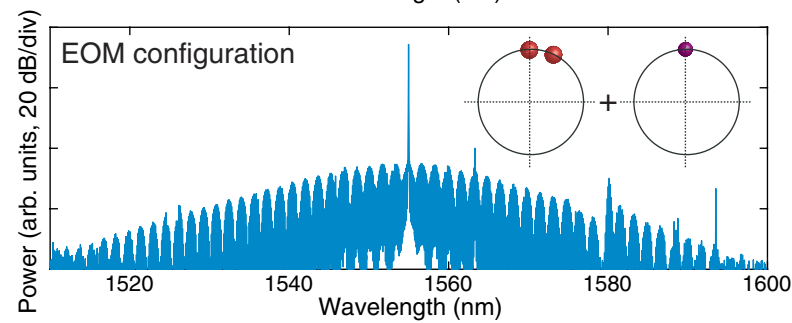

FIG. 12. Experimental realization of single-cavity-resonance excitation with dual pumping. (a) Two experimental configurations we use to pump two dissimilar soliton microcombs from the same cavity resonance. (b) The microcomb spectrum generated with the dual-laser configuration. The constituent soliton pattern is illustrated in the inset. (c) The microcomb spectrum generated with the one-laser-with-EOM configuration.

observed, indicating that close-range binding is formed. With the electro-optical sampling technique we are able to confirm that the intersoliton separations are of a few hundred femtoseconds, which are similar to those of the dual-pumped composite solitons. A comprehensive study of the coexistence of two soliton species excited in a single cavity resonance can be found in Ref. [41].

[1] A. Hasegawa, Optical Solitons in Fibers (Springer, New York, 1989), pp. 1-74.

[2] Y.S. Kivshar and G. Agrawal, Optical Solitons: From Fibers to Photonic Crystals (Academic Press, New York, 2003).

[3] N. Akhmediev and A. Ankiewicz, Dissipative Solitons in the Complex Ginzburg-Landau and Swift-Hohenberg Equations, in Dissipative Solitons (Springer, New York, (2005), pp. $1-17$.

[4] N. Lilienfein, C. Hofer, M. Högner, T. Saule, M. Trubetskov, V. Pervak, E. Fill, C. Riek, A. Leitenstorfer, J. Limpert et al., Temporal Solitons in Free-Space Femtosecond Enhancement Cavities, Nat. Photonics 13, 214 (2019).

[5] F. Leo, S. Coen, P. Kockaert, S.-P. Gorza, P. Emplit, and M. Haelterman, Temporal Cavity Solitons in One-Dimensional Kerr Media as Bits in an All-Optical Buffer, Nat. Photonics 4, 471 (2010). 
[6] T. Herr, V. Brasch, J. D. Jost, C. Y. Wang, N. M. Kondratiev, M. L. Gorodetsky, and T. J. Kippenberg, Temporal Solitons in Optical Microresonators, Nat. Photonics 8, 145 (2014).

[7] T. J. Kippenberg, A. L. Gaeta, M. Lipson, and M. L. Gorodetsky, Dissipative Kerr Solitons in Optical Microresonators, Science 361, eaan8083 (2018).

[8] A. Dutt, C. Joshi, X. Ji, J. Cardenas, Y. Okawachi, K. Luke, A. L. Gaeta, and M. Lipson, On-Chip Dual-Comb Source for Spectroscopy, Sci. Adv. 4, e1701858 (2018).

[9] E. Obrzud, M. Rainer, A. Harutyunyan, M. H. Anderson, J. Liu, M. Geiselmann, B. Chazelas, S. Kundermann, S. Lecomte, M. Cecconi, A. Ghedina, E. Molinari, F. Pepe, F. Wildi, F. Bouchy, T. J. Kippenberg, and T. Herr, A Microphotonic Astrocomb, Nat. Photonics 13, 31 (2019).

[10] W. Liang, D. Eliyahu, V. S. Ilchenko, A. A. Savchenkov, A. B. Matsko, D. Seidel, and L. Maleki, High Spectral Purity Kerr Frequency Comb Radio Frequency Photonic Oscillator, Nat. Commun. 6, 7957 (2015).

[11] W. Weng, E. Lucas, G. Lihachev, V. E. Lobanov, H. Guo, M. L. Gorodetsky, and T. J. Kippenberg, Spectral Purification of Microwave Signals with Disciplined Dissipative Kerr Solitons, Phys. Rev. Lett. 122, 013902 (2019).

[12] C. Bao, M.-G. Suh, and K. Vahala, Microresonator Soliton Dual-Comb Imaging, Optica 6, 1110 (2019).

[13] P. Marin-Palomo, J. N. Kemal, M. Karpov, A. Kordts, J. Pfeifle, M. H. P. Pfeiffer, P. Trocha, S. Wolf, V. Brasch, M. H. Anderson et al., Microresonator-Based Solitons for Massively Parallel Coherent Optical Communications, Nature (London) 546, 274 (2017).

[14] Q.-F. Yang, X. Yi, K. Y. Yang, and K. Vahala, Stokes Solitons in Optical Microcavities, Nat. Phys. 13, 53 (2017).

[15] C. Bao, J. A. Jaramillo-Villegas, Y. Xuan, D. E. Leaird, M. Qi, and A. M. Weiner, Observation of Fermi-Pasta-Ulam Recurrence Induced by Breather Solitons in an Optical Microresonator, Phys. Rev. Lett. 117, 163901 (2016).

[16] M. Yu, J. K. Jang, Y. Okawachi, A. G. Griffith, K. Luke, S. A. Miller, X. Ji, M. Lipson, and A. L. Gaeta, Breather Soliton Dynamics in Microresonators, Nat. Commun. 8, 14569 (2017).

[17] E. Lucas, M. Karpov, H. Guo, M. L. Gorodetsky, and T. J. Kippenberg, Breathing Dissipative Solitons in Optical Microresonators, Nat. Commun. 8, 736 (2017).

[18] D. C. Cole, E. S. Lamb, P. Del'Haye, S. A. Diddams, and S. B. Papp, Soliton Crystals in Kerr Resonators, Nat. Photonics 11, 671 (2017).

[19] M. Stratmann, T. Pagel, and F. Mitschke, Experimental Observation of Temporal Soliton Molecules, Phys. Rev. Lett. 95, 143902 (2005).

[20] A. Hause, H. Hartwig, M. Böhm, and F. Mitschke, Binding Mechanism of Temporal Soliton Molecules, Phys. Rev. A 78, 063817 (2008).

[21] M. Olivier, V. Roy, M. Piché, and F. Babin, Pulse Collisions in the Stretched-Pulse Fiber Laser, Opt. Lett. 29, 1461 (2004).

[22] V. Roy, M. Olivier, F. Babin, and M. Piché, Dynamics of Periodic Pulse Collisions in a Strongly DissipativeDispersive System, Phys. Rev. Lett. 94, 203903 (2005).

[23] Q.-F. Yang, B. Shen, H. Wang, M. Tran, Z. Zhang, K. Y. Yang, L. Wu, C. Bao, J. Bowers, A. Yariv et al.,
Vernier Spectrometer Using Counterpropagating Soliton Microcombs, Science 363, 965 (2019).

[24] B. Lomsadze, B.C. Smith, and S. T. Cundiff, Tri-Comb Spectroscopy, Nat. Photonics 12, 676 (2018).

[25] E. Lucas, G. Lihachev, R. Bouchand, N. G. Pavlov, A. S. Raja, M. Karpov, M. L. Gorodetsky, and T. J. Kippenberg, Spatial Multiplexing of Soliton Microcombs, Nat. Photonics 12, 699 (2018).

[26] K. Goda and B. Jalali, Dispersive Fourier Transformation for Fast Continuous Single-Shot Measurements, Nat. Photonics 7, 102 (2013).

[27] X. Yi, Q.-F. Yang, K. Y. Yang, and K. Vahala, Imaging Soliton Dynamics in Optical Microcavities, Nat. Commun. 9, 3565 (2018).

[28] T. Herr, V. Brasch, J. D. Jost, I. Mirgorodskiy, G. Lihachev, M. L. Gorodetsky, and T. J. Kippenberg, Mode Spectrum and Temporal Soliton Formation in Optical Microresonators, Phys. Rev. Lett. 113, 123901 (2014).

[29] P. Parra-Rivas, D. Gomila, P. Colet, and L. Gelens, Interaction of Solitons and the Formation of Bound States in the Generalized Lugiato-Lefever Equation, Eur. Phys. J. D 71, 198 (2017).

[30] Y. Wang, F. Leo, J. Fatome, M. Erkintalo, S. G. Murdoch, and S. Coen, Universal Mechanism for the Binding of Temporal Cavity Solitons, Optica 4, 855 (2017).

[31] A. G. Vladimirov, S. V. Gurevich, and M. Tlidi, Effect of Cherenkov Radiation on Localized-State Interaction, Phys. Rev. A 97, 013816 (2018).

[32] W. Weng, R. Bouchand, E. Lucas, and T. J. Kippenberg, Polychromatic Cherenkov Radiation Induced Group Velocity Symmetry Breaking in Counterpropagating Dissipative Kerr Solitons, Phys. Rev. Lett. 123, 253902 (2019).

[33] K. Luo, J. K. Jang, S. Coen, S. G. Murdoch, and M. Erkintalo, Spontaneous Creation and Annihilation of Temporal Cavity Solitons in a Coherently Driven Passive Fiber Resonator, Opt. Lett. 40, 3735 (2015).

[34] H. Taheri, A. A. Eftekhar, K. Wiesenfeld, and A. Adibi, Soliton Formation in Whispering-Gallery-Mode Resonators via Input Phase Modulation, IEEE Photonics J. 7, 1 (2015).

[35] J. K. Jang, M. Erkintalo, K. Luo, G.-L. Oppo, S. Coen, and S. G. Murdoch, Controlled Merging and Annihilation of Localised Dissipative Structures in an ac-Driven Damped Nonlinear Schrödinger System, New J. Phys. 18, 033034 (2016).

[36] V.E. Lobanov, G. V. Lihachev, N. G. Pavlov, A. V. Cherenkov, T. J. Kippenberg, and M. L. Gorodetsky, Harmonization of Chaos into a Soliton in Kerr Frequency Combs, Opt. Express 24, 27382 (2016).

[37] F. Treussart, V. S. Ilchenko, J.-F. Roch, J. Hare, V. LefevreSeguin, J.-M. Raimond, and S. Haroche, Evidence for Intrinsic Kerr Bistability of High-Q Microsphere Resonators in Superfluid Helium, Eur. Phys. J. 1, 235 (1998).

[38] S. Coen and M. Erkintalo, Universal Scaling Laws of Kerr Frequency Combs, Opt. Lett. 38, 1790 (2013).

[39] T. Hansson and S. Wabnitz, Frequency Comb Generation Beyond the Lugiato-Lefever Equation: Multi-Stability and Super Cavity Solitons, J. Opt. Soc. Am. B 32, 1259 (2015).

[40] M. Anderson, Y. Wang, F. Leo, S. Coen, M. Erkintalo, and S. G. Murdoch, Coexistence of Multiple Nonlinear States in 
a Tristable Passive Kerr Resonator, Phys. Rev. X 7, 031031 (2017).

[41] W. Weng, R. Bouchand, E. Lucas, E. Obrzud, T. Herr, and T. Kippenberg, Heteronuclear Soliton Molecules in Optical Microresonators, arXiv:1901.04026.

[42] A. U. Nielsen, B. Garbin, S. Coen, S. G. Murdoch, and M. Erkintalo, Coexistence and Interactions between Nonlinear States with Different Polarizations in a Monochromatically Driven Passive Kerr Resonator, Phys. Rev. Lett. 123, 013902 (2019).

[43] G. I. Stegeman and M. Segev, Optical Spatial Solitons and Their Interactions: Universality and Diversity, Science 286, 1518 (1999).

[44] T. Hansson and S. Wabnitz, Bichromatically Pumped Microresonator Frequency Combs, Phys. Rev. A 90, 013811 (2014).

[45] I. Hendry, W. Chen, Y. Wang, B. Garbin, J. Javaloyes, G.-L. Oppo, S. Coen, S. G. Murdoch, and M. Erkintalo, Spontaneous Symmetry Breaking and Trapping of Temporal Kerr Cavity Solitons by Pulsed or Amplitude-Modulated Driving Fields, Phys. Rev. A 97, 053834 (2018).

[46] E. Obrzud, S. Lecomte, and T. Herr, Temporal Solitons in Microresonators Driven by Optical Pulses, Nat. Photonics 11, 600 (2017).

[47] S. Coen, M. Tlidi, P. Emplit, and M. Haelterman, Convection versus Dispersion in Optical Bistability, Phys. Rev. Lett. 83, 2328 (1999).

[48] D. Ceoldo, A. Bendahmane, J. Fatome, G. Millot, T. Hansson, D. Modotto, S. Wabnitz, and B. Kibler, Multiple Four-Wave Mixing and Kerr Combs in a Bichromatically Pumped Nonlinear Fiber Ring Cavity, Opt. Lett. 41, 5462 (2016).

[49] A. Bendahmane, J. Fatome, C. Finot, G. Millot, and B. Kibler, Selective Generation of Kerr Combs Induced by Asymmetrically Phase-Detuned Dual Pumping of a Fiber Ring Cavity, Opt. Lett. 43, 4449 (2018).

[50] H. Guo, E. Lucas, M. H. P. Pfeiffer, M. Karpov, M. Anderson, J. Liu, M. Geiselmann, J. D. Jost, and T. J. Kippenberg, Intermode Breather Solitons in Optical Microresonators, Phys. Rev. X 7, 041055 (2017).

[51] L. A. Lugiato and R. Lefever, Spatial Dissipative Structures in Passive Optical Systems, Phys. Rev. Lett. 58, 2209 (1987).

[52] M. Haelterman, S. Trillo, and S. Wabnitz, Dissipative Modulation Instability in a Nonlinear Dispersive Ring Cavity, Opt. Commun. 91, 401 (1992).

[53] See Supplemental Material at http://link.aps.org/ supplemental/10.1103/PhysRevX.10.021017 for additional analysis, experimental results, and corroborating simulations, which also includes Refs. [6,11,44,45,54-65].

[54] J. K. Jang, M. Erkintalo, S. Coen, and S. G. Murdoch, Temporal Tweezing of Light through the Trapping and Manipulation of Temporal Cavity Solitons, Nat. Commun. 6, 7370 (2015).

[55] J. Javaloyes, P. Camelin, M. Marconi, and M. Giudici, Dynamics of Localized Structures in Systems with Broken Parity Symmetry, Phys. Rev. Lett. 116, 133901 (2016).

[56] P. Parra-Rivas, D. Gomila, M. A. Matías, P. Colet, and L. Gelens, Effects of Inhomogeneities and Drift on the
Dynamics of Temporal Solitons in Fiber Cavities and Microresonators, Opt. Express 22, 30943 (2014).

[57] J. K. Jang, A. Klenner, X. Ji, Y. Okawachi, M. Lipson, and A. L. Gaeta, Synchronization of Coupled Optical Microresonators, Nat. Photonics 12, 688 (2018).

[58] G. I. Stegeman and M. Segev, Optical Spatial Solitons and Their Interactions: Universality and Diversity, Science 286, 1518 (1999).

[59] C. R. Menyuk, Stability of Solitons in Birefringent Optical Fibers. I: Equal Propagation Amplitudes, Opt. Lett. 12, 614 (1987).

[60] M. N. Islam, C. D. Poole, and J. P. Gordon, Soliton Trapping in Birefringent Optical Fibers, Opt. Lett. 14, 1011 (1989).

[61] B. C. Collings, S. T. Cundiff, N. N. Akhmediev, J. M. Soto-Crespo, K. Bergman, and W. H. Knox, PolarizationLocked Temporal Vector Solitons in a Fiber Laser: Experiment, J. Opt. Soc. Am. B 17, 354 (2000).

[62] J. K. Jang, M. Erkintalo, S. G. Murdoch, and S. Coen, Ultraweak Long-Range Interactions of Solitons Observed Over Astronomical Distances, Nat. Photonics 7, 657 (2013).

[63] M. Pang, W. He, X. Jiang, and P. S. J. Russell, All-Optical Bit Storage in a Fibre Laser by Optomechanically Bound States of Solitons, Nat. Photonics 10, 454 (2016).

[64] B. W. Plansinis, W. R. Donaldson, and G. P. Agrawal, What Is the Temporal Analog of Reflection and Refraction of Optical Beams?, Phys. Rev. Lett. 115, 183901 (2015).

[65] B. Plansinis, Applications of Space-Time Duality, Ph.D. Thesis, University of Rochester, 2017.

[66] H. Guo, M. Karpov, E. Lucas, A. Kordts, M. H. P. Pfeiffer, V. Brasch, G. Lihachev, V. E. Lobanov, M. L. Gorodetsky, and T. J. Kippenberg, Universal Dynamics and Deterministic Switching of Dissipative Kerr Solitons in Optical Microresonators, Nat. Phys. 13, 94 (2017).

[67] A. B. Matsko and L. Maleki, Noise Conversion in Kerr Comb rf Photonic Oscillators, J. Opt. Soc. Am. B 32, 232 (2015).

[68] A. B. Matsko and L. Maleki, Feshbach Resonances in Kerr Frequency Combs, Phys. Rev. A 91, 013831 (2015).

[69] Q.-F. Yang, X. Yi, K. Y. Yang, and K. Vahala, Spatial-ModeInteraction-Induced Dispersive Waves and Their Active Tuning in Microresonators, Optica 3, 1132 (2016).

[70] C. Bao, Y. Xuan, C. Wang, J. A. Jaramillo-Villegas, D. E. Leaird, M. Qi, and A. M. Weiner, Soliton Repetition Rate in a Silicon-Nitride Microresonator, Opt. Lett. 42, 759 (2017).

[71] A. V. Cherenkov, V. E. Lobanov, and M. L. Gorodetsky, Dissipative Kerr Solitons and Cherenkov Radiation in Optical Microresonators with Third-Order Dispersion, Phys. Rev. A 95, 033810 (2017).

[72] H. Taheri, A. B. Matsko, and L. Maleki, Optical Lattice Trap for Kerr Solitons, Eur. Phys. J. D 71, 153 (2017).

[73] R. J. Weiblen and I. Vurgaftman, Bichromatic Pumping in Mid-Infrared Microresonator Frequency Combs with Higher-Order Dispersion, Opt. Express 27, 4238 (2019).

[74] T. G. Philbin, C. Kuklewicz, S. Robertson, S. Hill, F. König, and U. Leonhardt, Fiber-Optical Analog of the Event Horizon, Science 319, 1367 (2008).

[75] S. Hill, C. E. Kuklewicz, U. Leonhardt, and F. König, Evolution of Light Trapped by a Soliton in a Microstructured Fiber, Opt. Express 17, 13588 (2009). 
[76] F. Belgiorno, S. L. Cacciatori, M. Clerici, V. Gorini, G. Ortenzi, L. Rizzi, E. Rubino, V. G. Sala, and D. Faccio, Hawking Radiation from Ultrashort Laser Pulse Filaments, Phys. Rev. Lett. 105, 203901 (2010).

[77] K. E. Webb, M. Erkintalo, Y. Xu, N. G. R. Broderick, J. M. Dudley, G. Genty, and S. G. Murdoch, Nonlinear Optics of Fibre Event Horizons, Nat. Commun. 5, 4969 (2014).

[78] B. W. Plansinis, W. R. Donaldson, and G. P. Agrawal, Single-Pulse Interference Caused by Temporal Reflection at Moving Refractive-Index Boundaries, J. Opt. Soc. Am. B 34, 2274 (2017).

[79] D. J. Kaup, B. A. Malomed, and J. Yang, Interchannel Pulse Collision in a Wavelength-Division-Multiplexed System with Strong Dispersion Management, Opt. Lett. 23, 1600 (1998).
[80] X. Wei, X. Liu, C. Xie, and L. F. Mollenauer, Reduction of Collision-Induced Timing Jitter in Dense WavelengthDivision Multiplexing by the Use of Periodic-GroupDelay Dispersion Compensators, Opt. Lett. 28, 983 (2003).

[81] X. Liu, X. Wei, L. F. Mollenauer, C. J. McKinstrie, and C. Xie, Collision-Induced Time Shift of a DispersionManaged Soliton and Its Minimization in WavelengthDivision-Multiplexed Transmission, Opt. Lett. 28, 1412 (2003).

[82] A. Del Duce, R. I. Killey, and P. Bayvel, Comparison of Nonlinear Pulse Interactions in 160-gb/s Quasi-Linear and Dispersion Managed Soliton Systems, J. Lightwave Technol. 22, 1263 (2004).

[83] See https://doi.org/10.5281/zenodo.3668712. 